\title{
Synthesis of 2-Sulfenylindenones via One-Pot Tandem Meyer-Schuster Rearrangement and Radical Cyclization of Arylpropynols with Disulfides
}

\author{
Xing-Song Zhang; Jun-Ying Jiao; Xiao-Hong Zhang;* Bo-Lun Hu and \\ Xing-Guo Zhang* \\ College of Chemistry and Materials Engineering, Wenzhou University, Wenzhou \\ 325035, China
}

$\underline{\text { kamenzxh@wzu.edu.cn; zxg@wzu.edu.cn }}$

\section{Supporting Information \\ List of Contents}

1. ${ }^{1} \mathrm{H}$ NMR and ${ }^{13} \mathrm{C}$ NMR of Compounds 3-29---------------------------S2-S57

2. X-Ray of compound 16---------------------------------------------------------S58-S59 
1. ${ }^{1}$ H NMR of 3-phenyl-2-(phenylthio)-1H-inden-1-one (3)

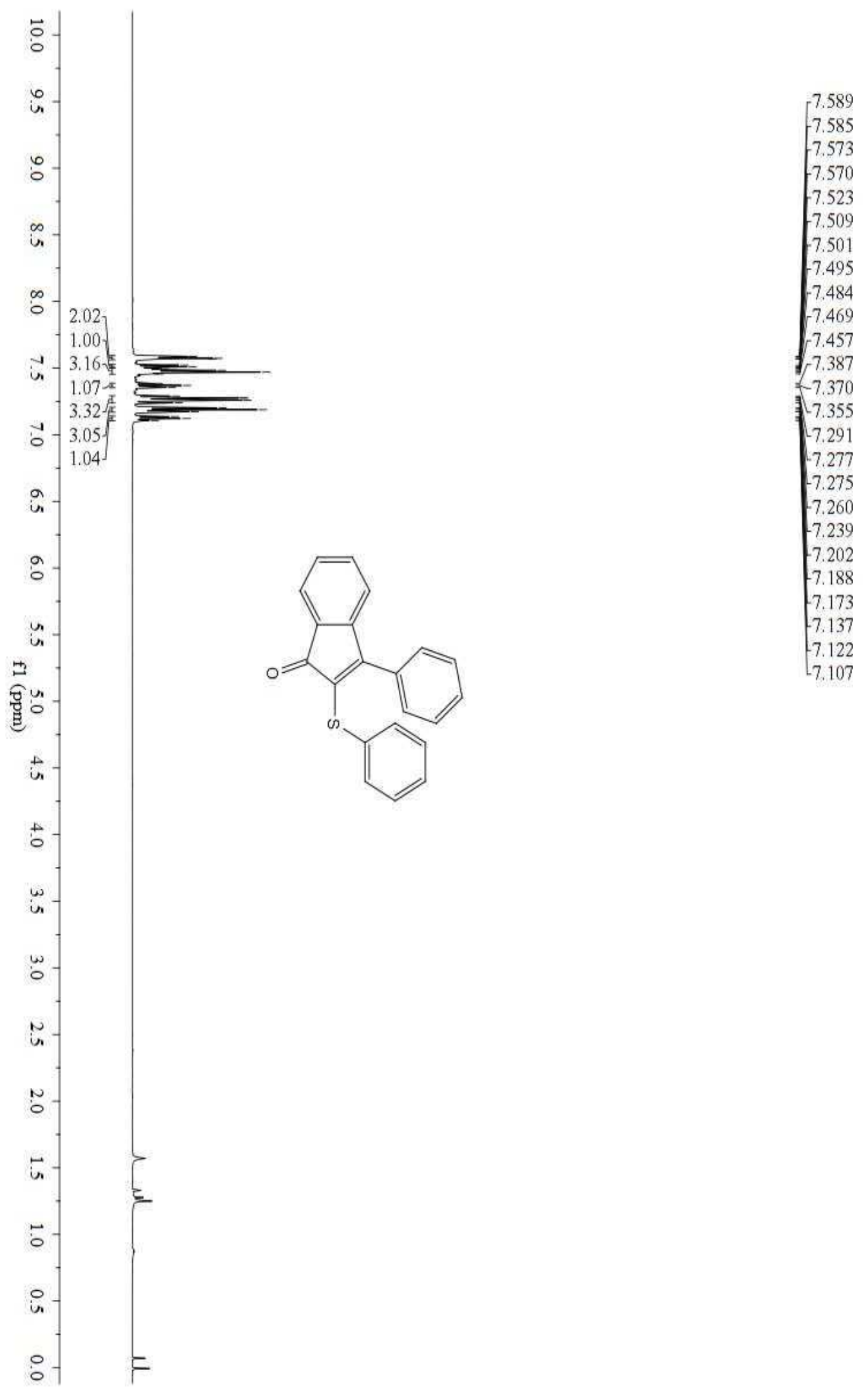




\section{${ }^{13}$ C NMR of 3-phenyl-2-(phenylthio)-1H-inden-1-one (3)}

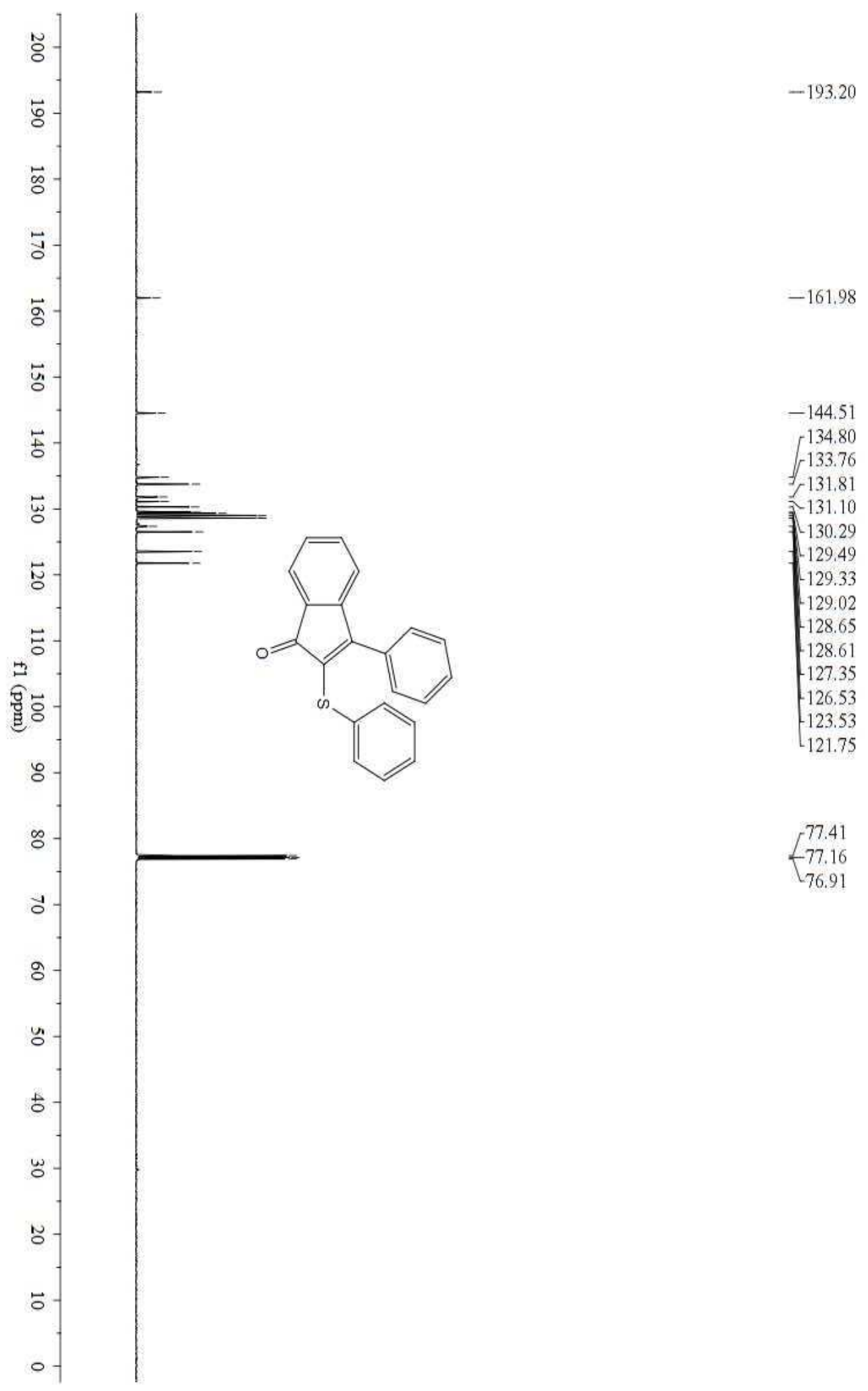


${ }^{1}$ H NMR of 3-phenyl-2-(p-tolylthio)-1H-inden-1-one (4)

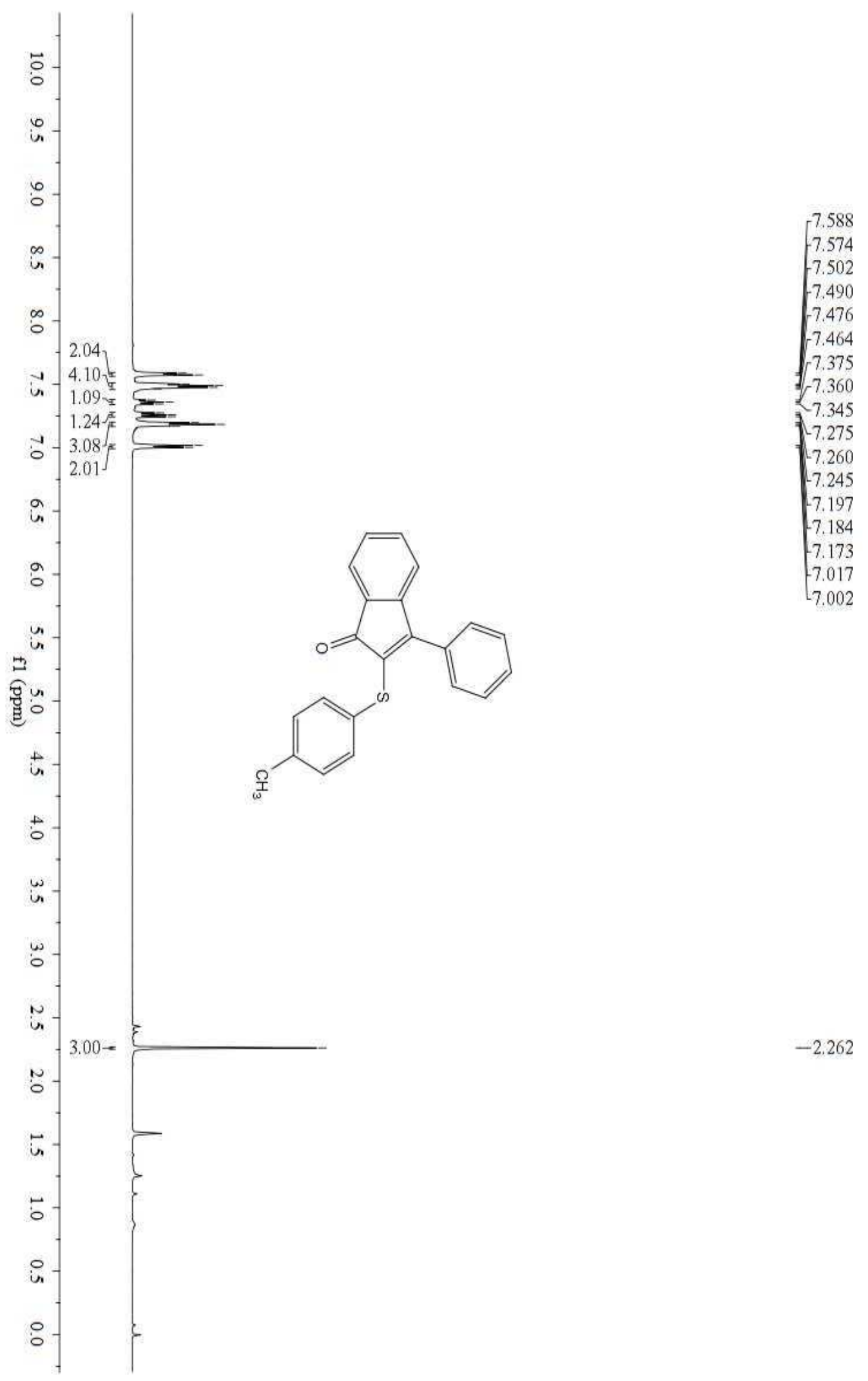




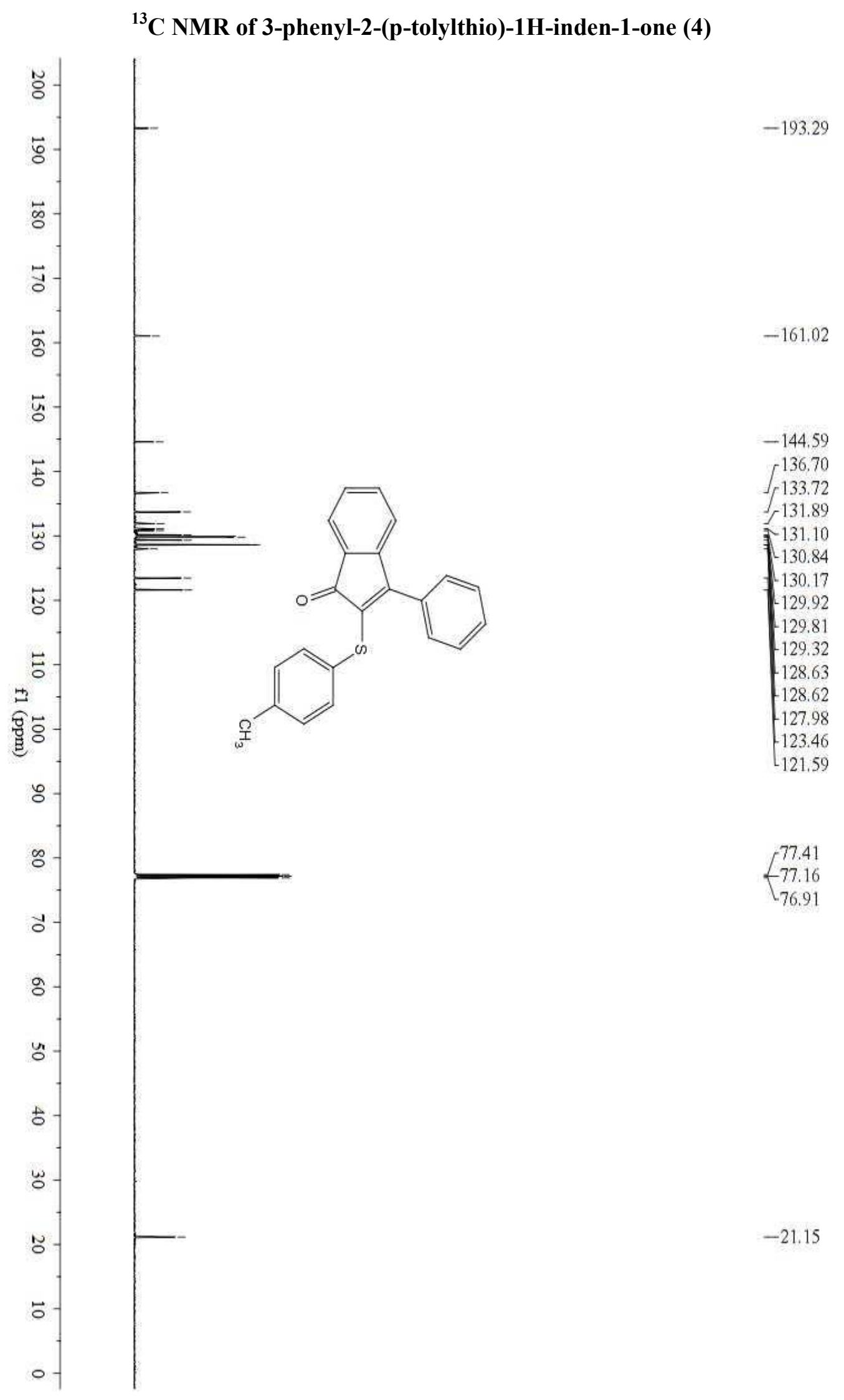


${ }^{1}$ H NMR of 2-((4-methoxyphenyl)thio)-3-phenyl-1H-inden-1-one (5)

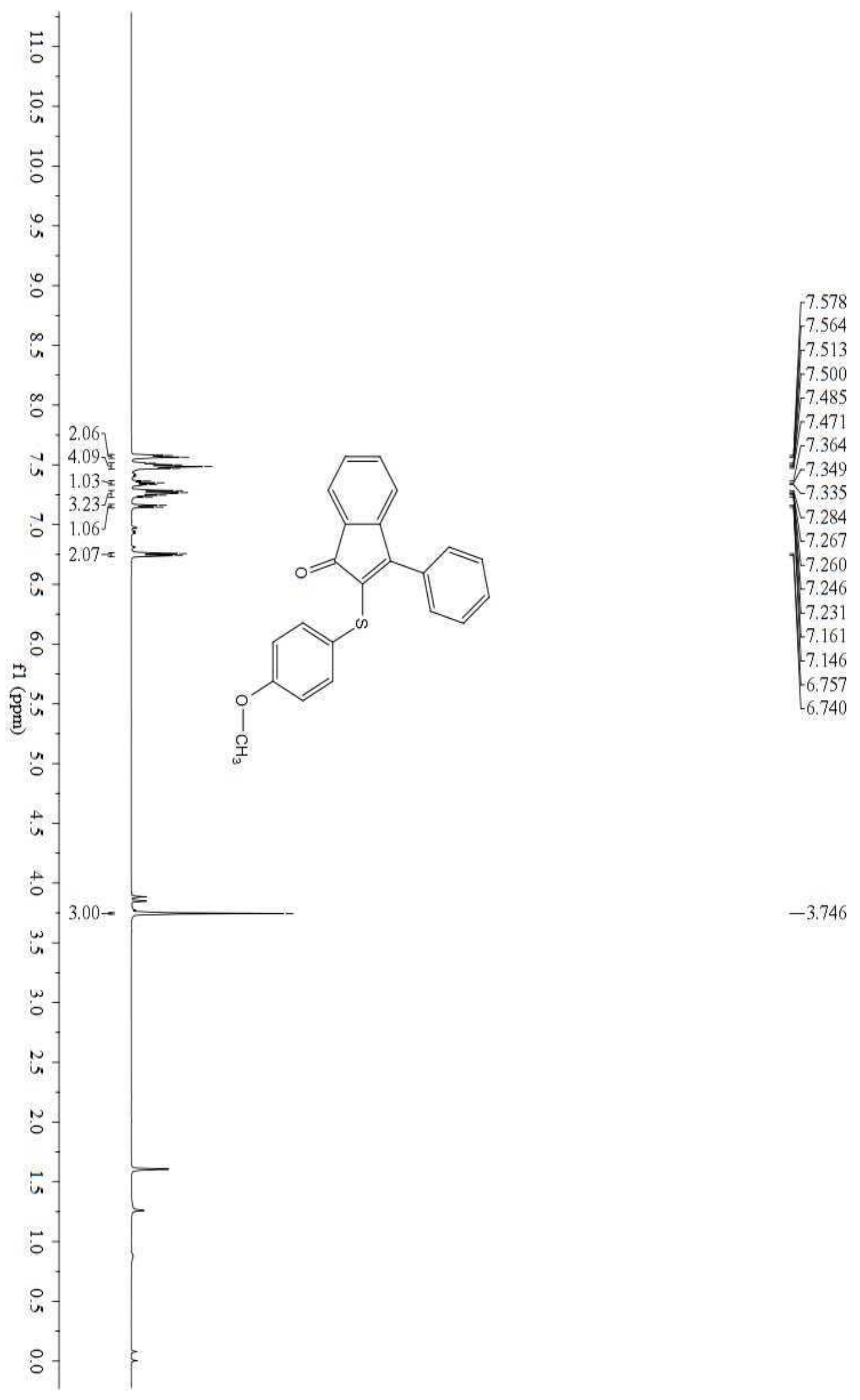




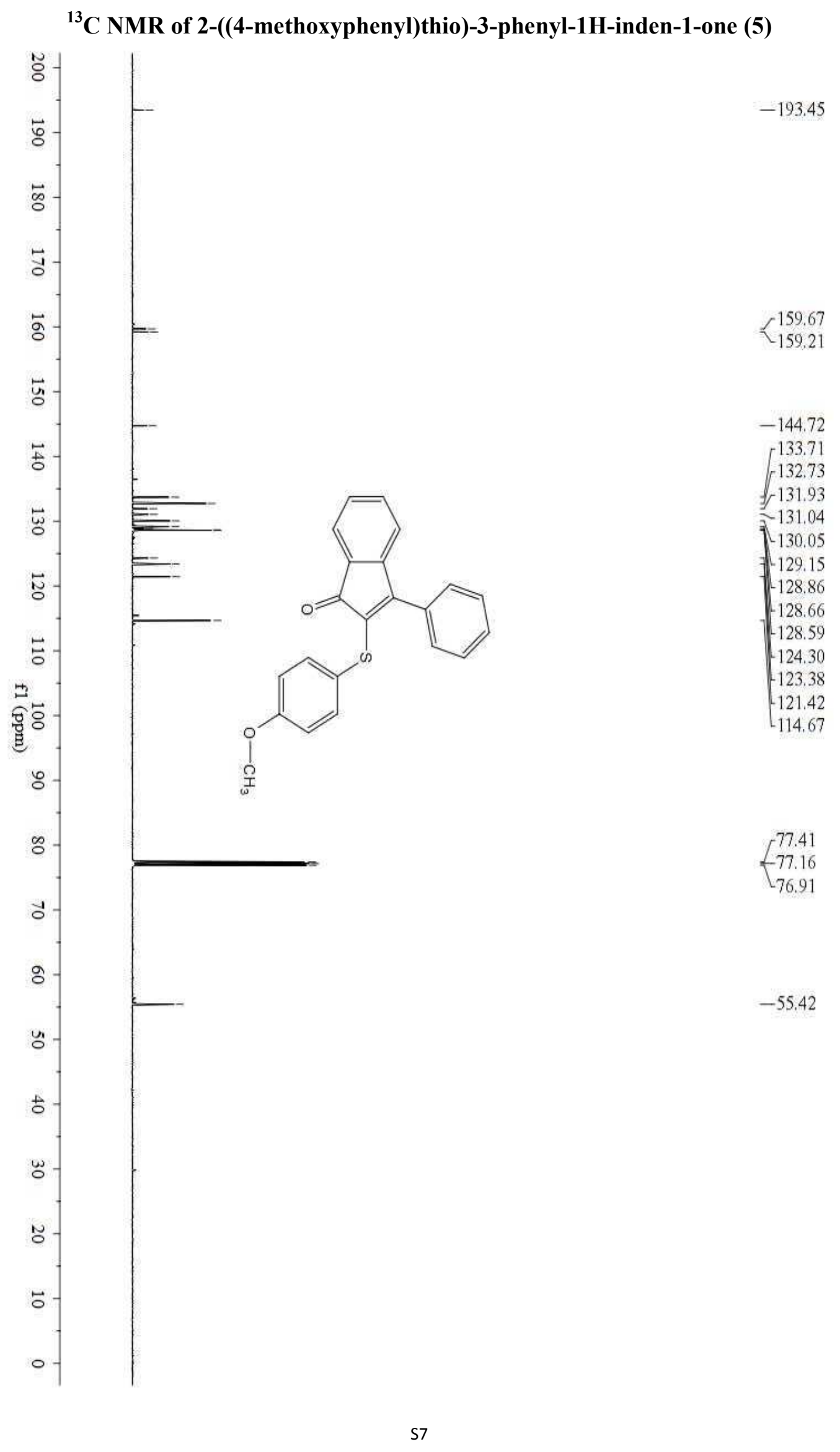


${ }^{1}$ H NMR of 2-((2-fluorophenyl)thio)-3-phenyl-1H-inden-1-one (6)

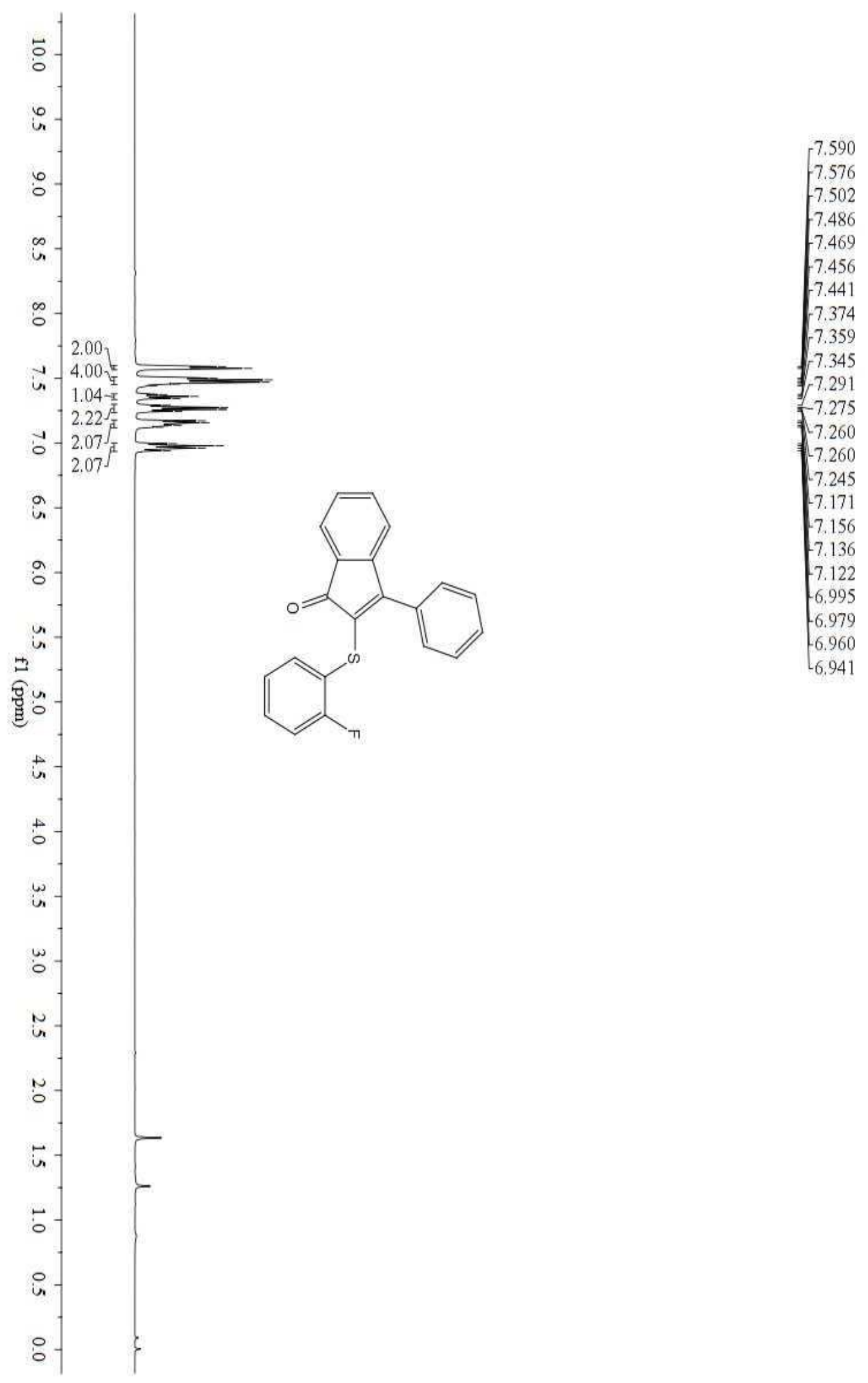




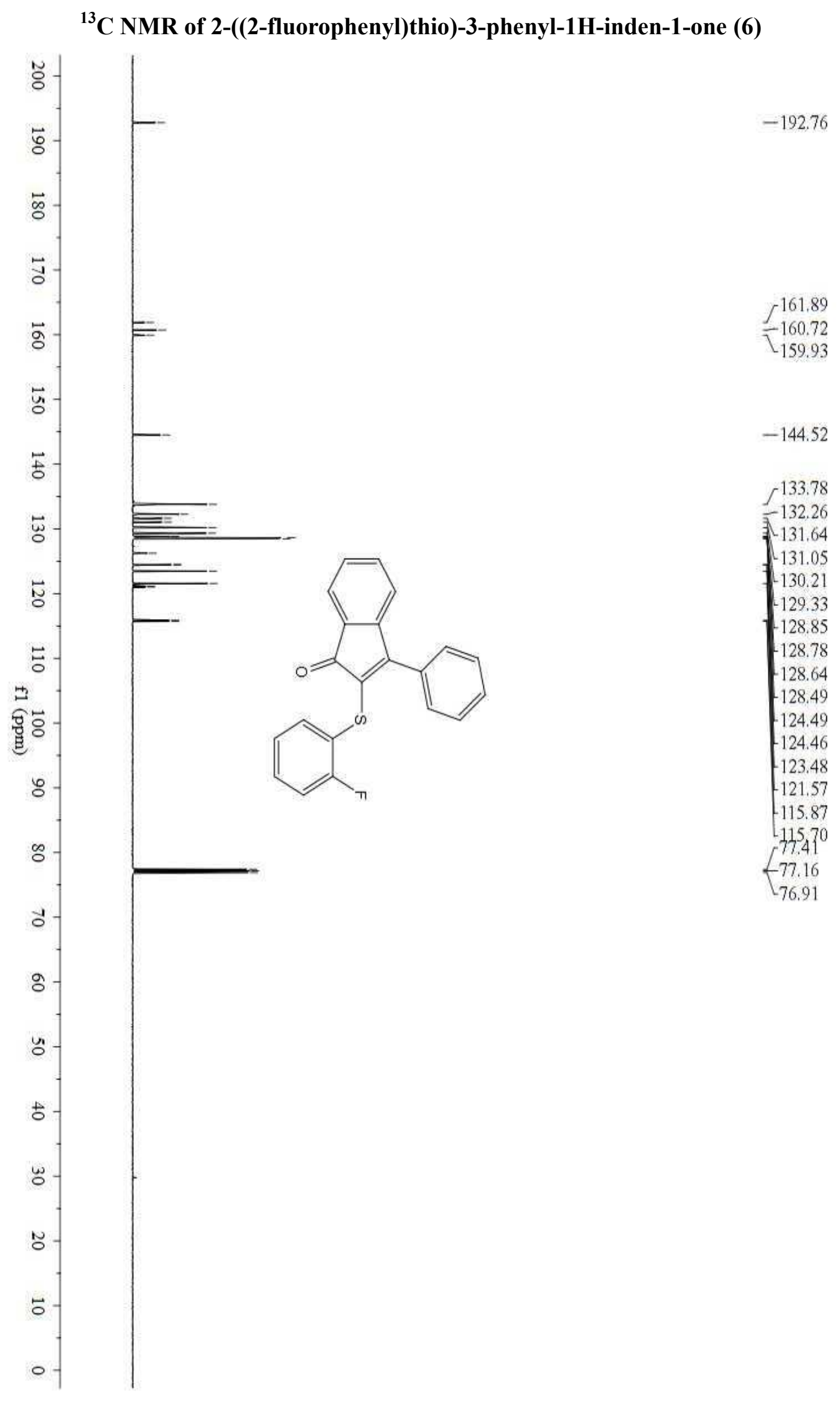


${ }^{1}$ H NMR of 2-((3-fluorophenyl)thio)-3-phenyl-1H-inden-1-one (7)

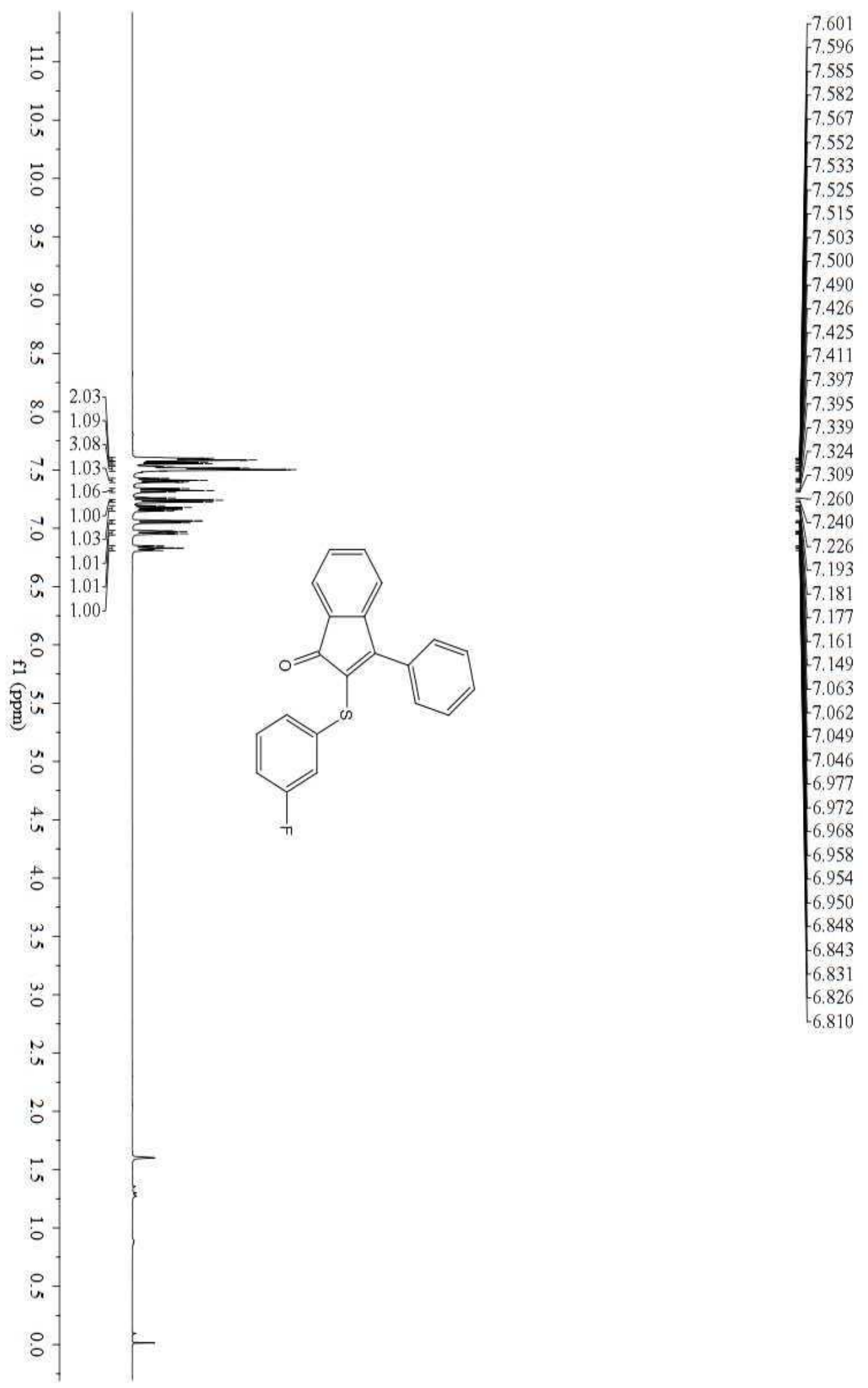


${ }^{13}$ C NMR of 2-((3-fluorophenyl)thio)-3-phenyl-1H-inden-1-one (7)

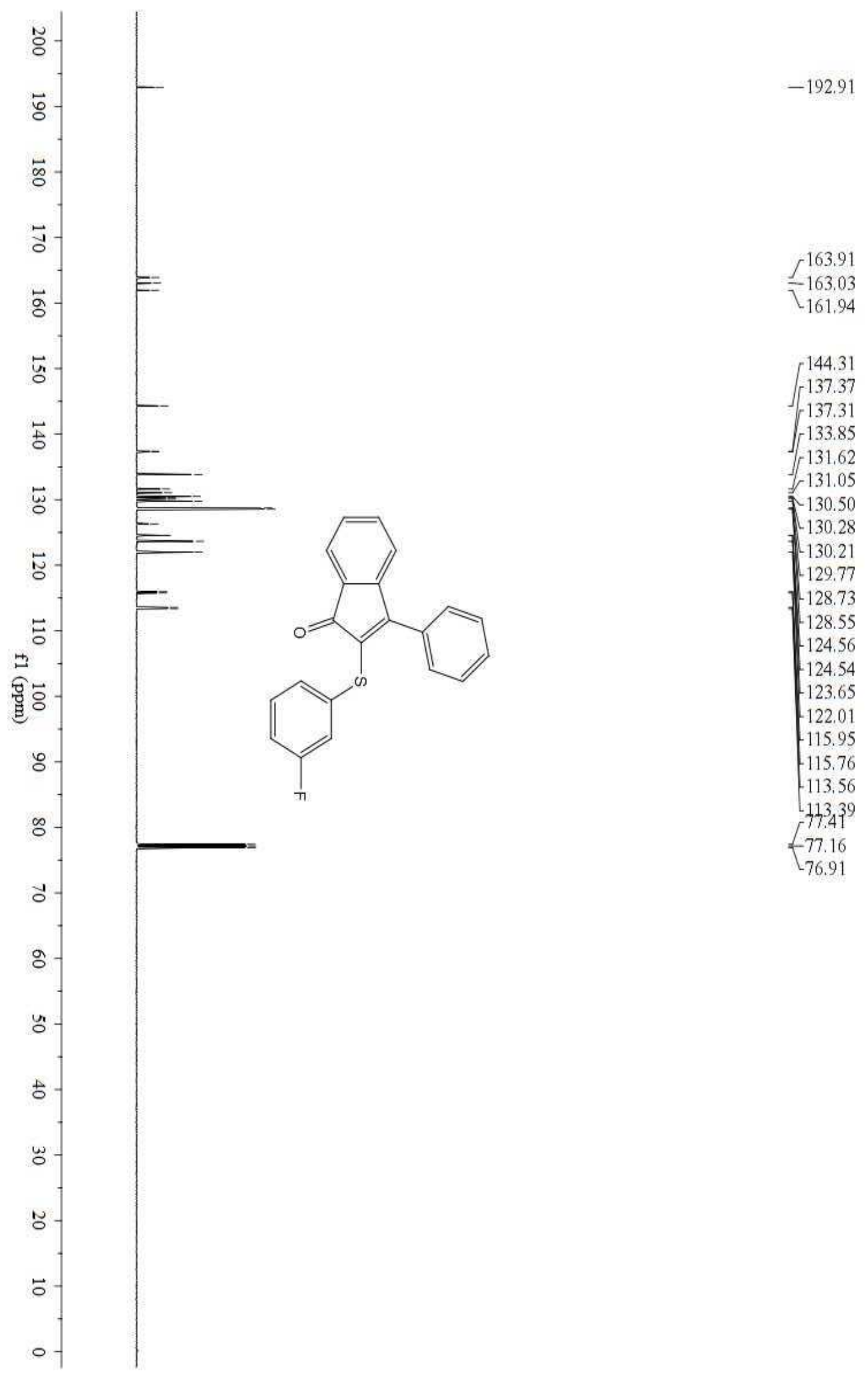


${ }^{1}$ H NMR of 2-((4-fluorophenyl)thio)-3-phenyl-1H-inden-1-one (8)

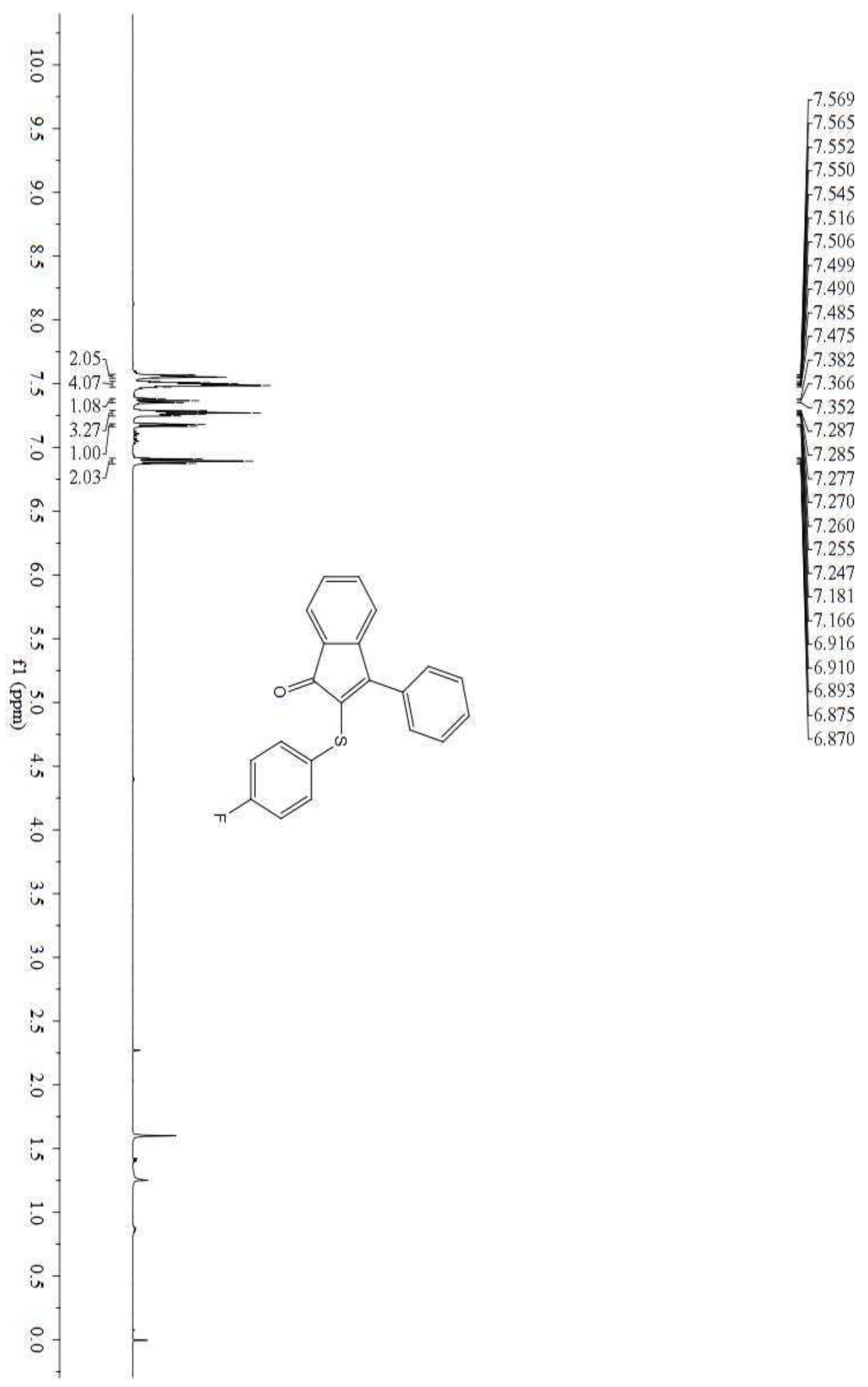


${ }^{13}$ C NMR of 2-((4-fluorophenyl)thio)-3-phenyl-1H-inden-1-one (8)

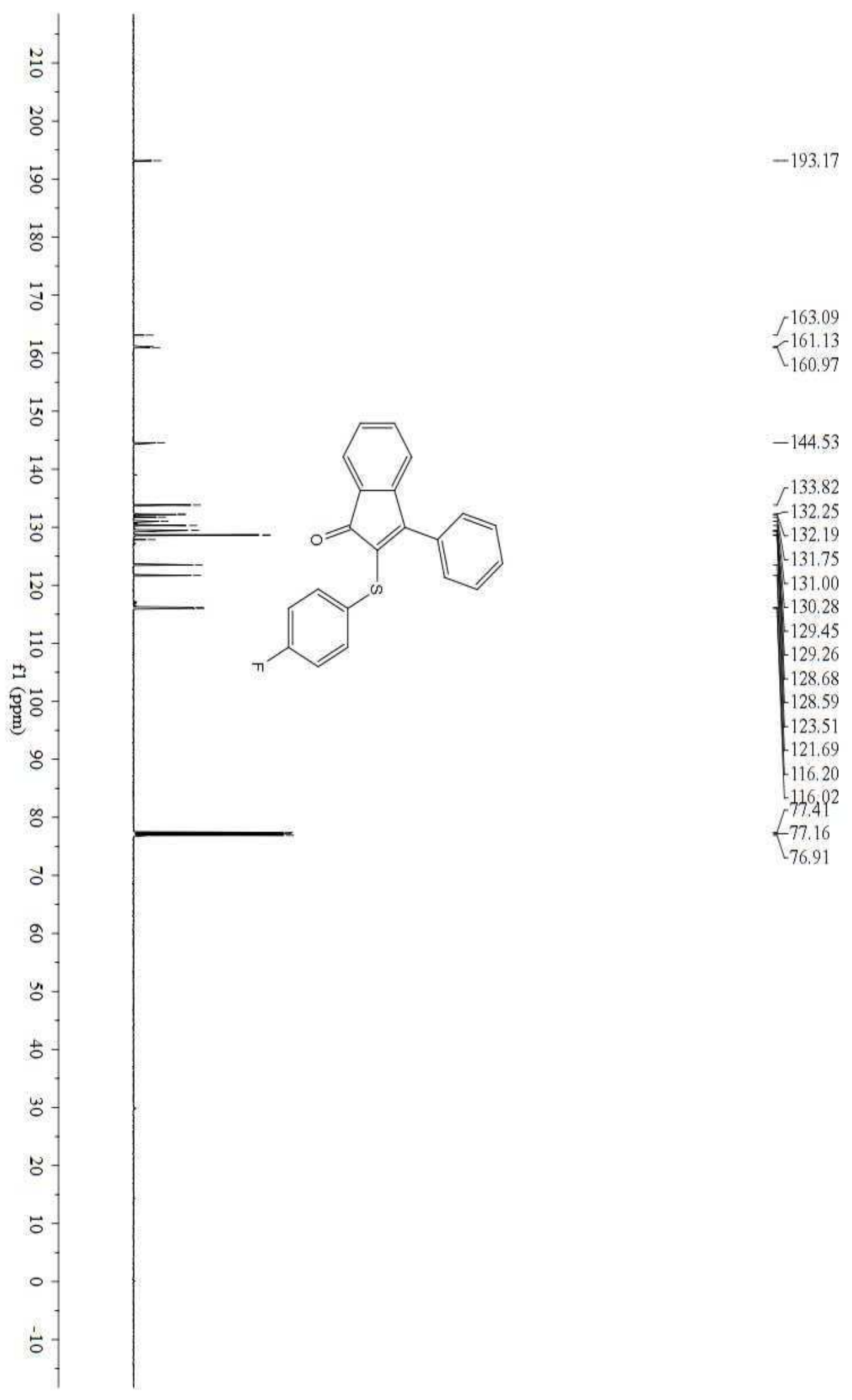




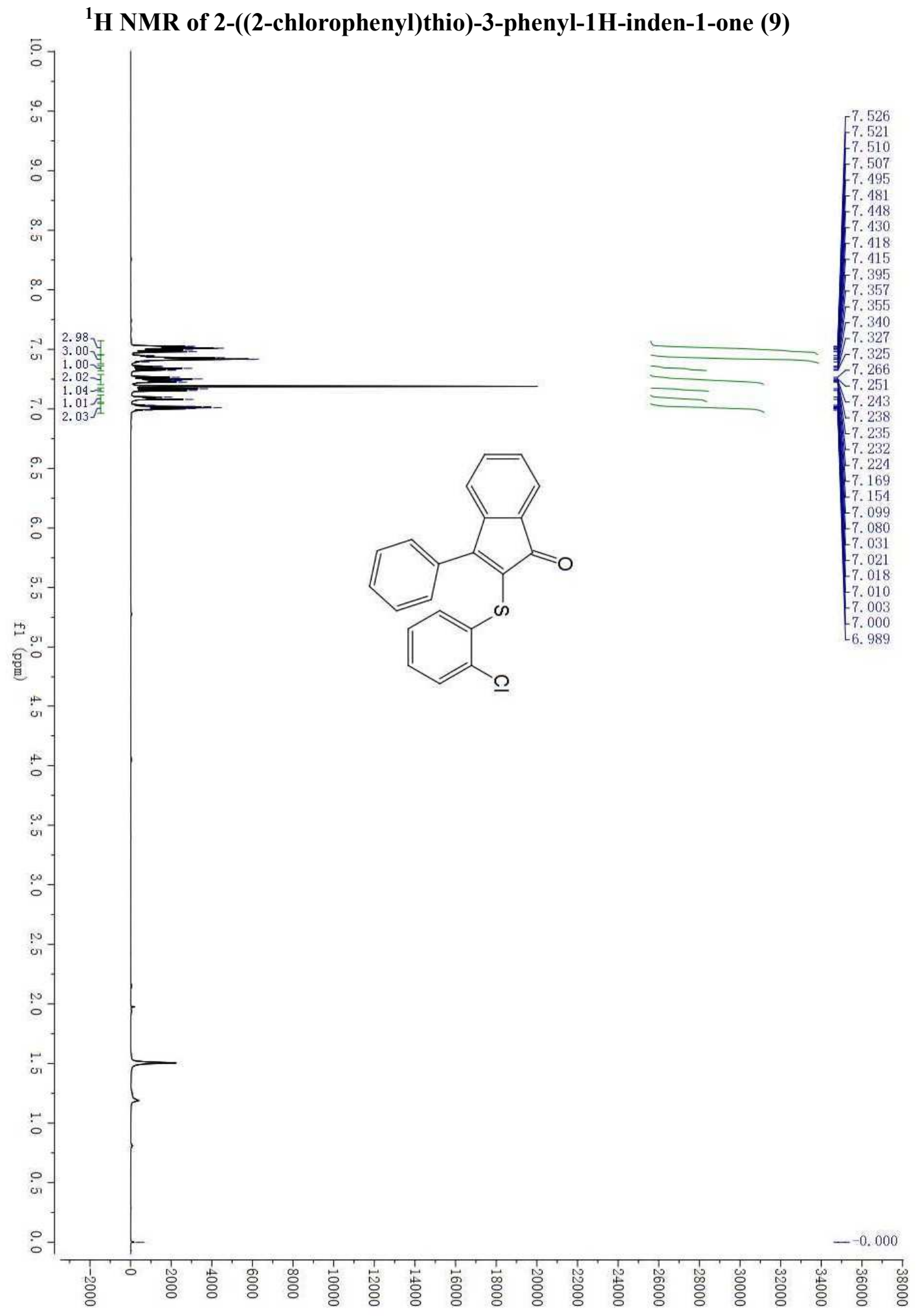


${ }^{13}$ C NMR of 2-((2-chlorophenyl)thio)-3-phenyl-1H-inden-1-one (9)

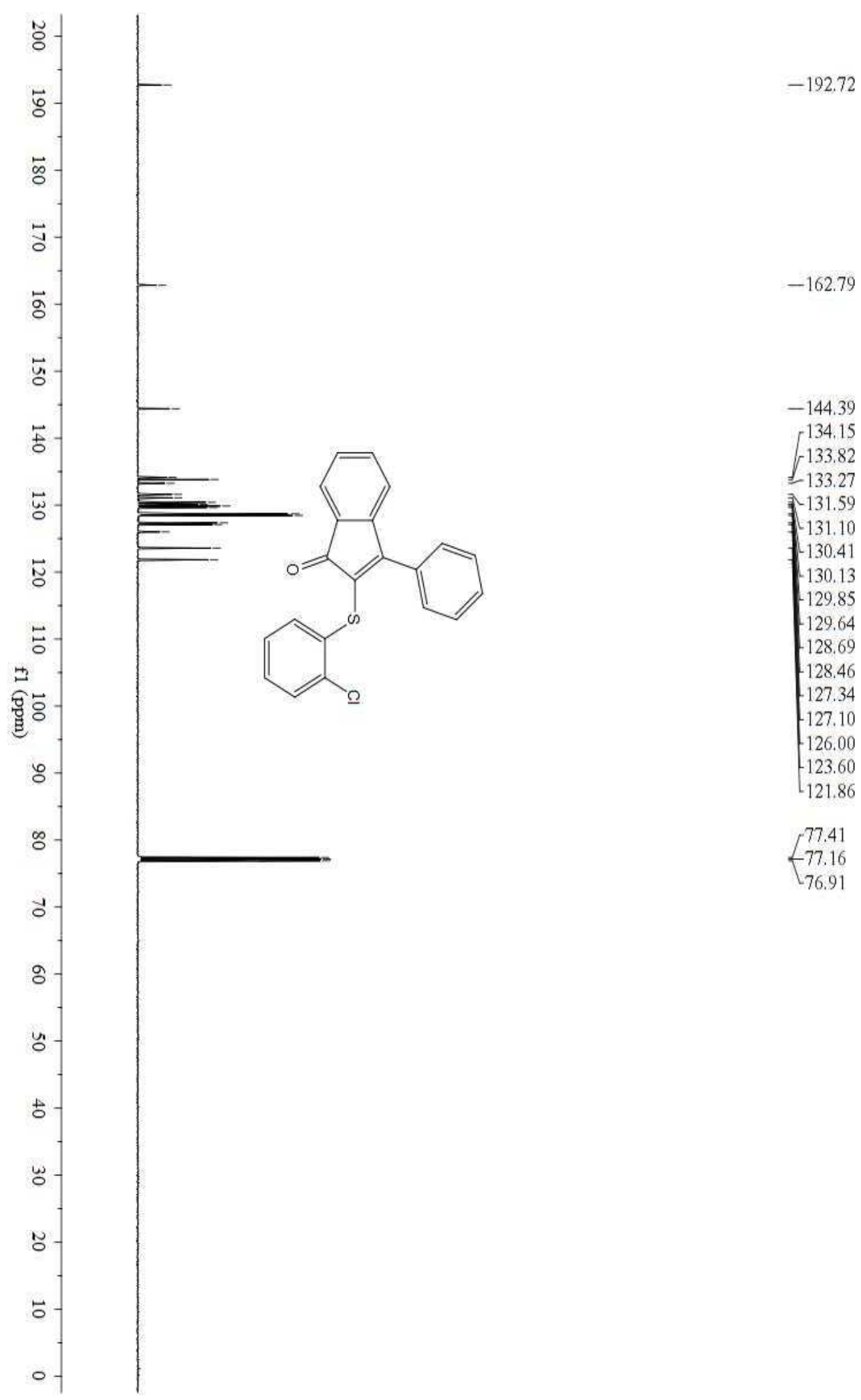


${ }^{1}$ H NMR of 2-((3-chlorophenyl)thio)-3-phenyl-1H-inden-1-one (10)

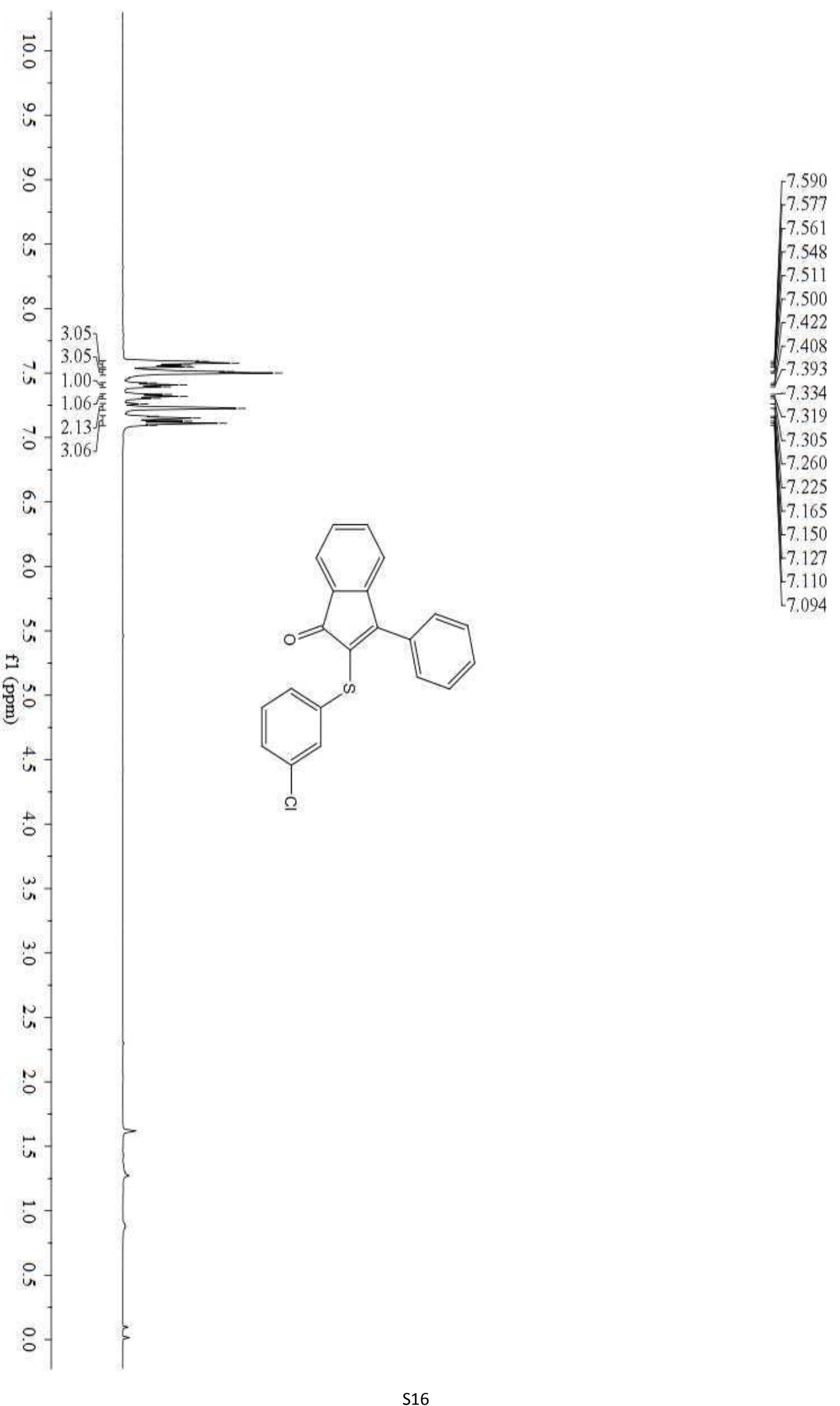


${ }^{13}$ C NMR of 2-((3-chlorophenyl)thio)-3-phenyl-1H-inden-1-one (10)

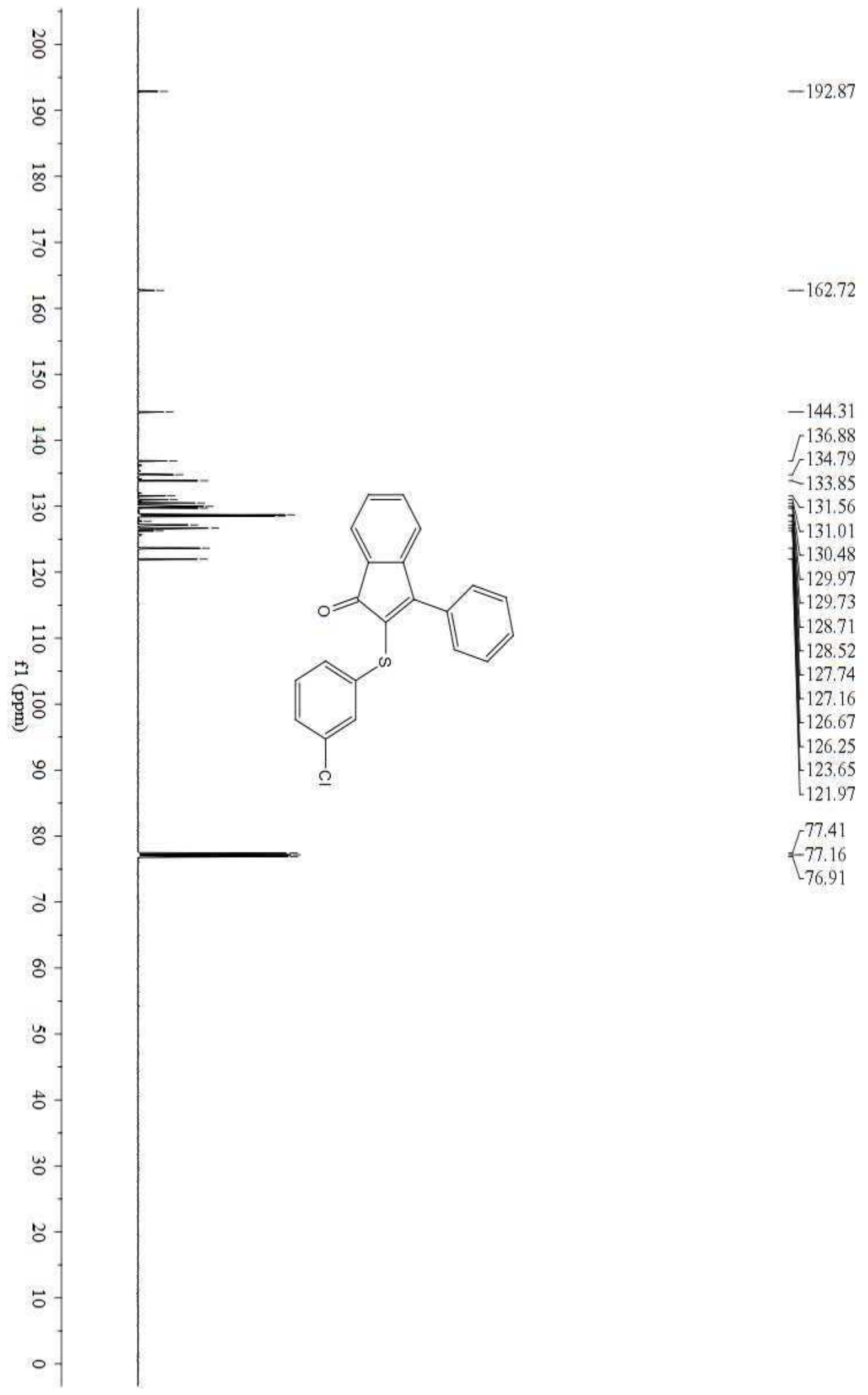


${ }^{1}$ H NMR of 2-((4-chlorophenyl)thio)-3-phenyl-1H-inden-1-one (11)

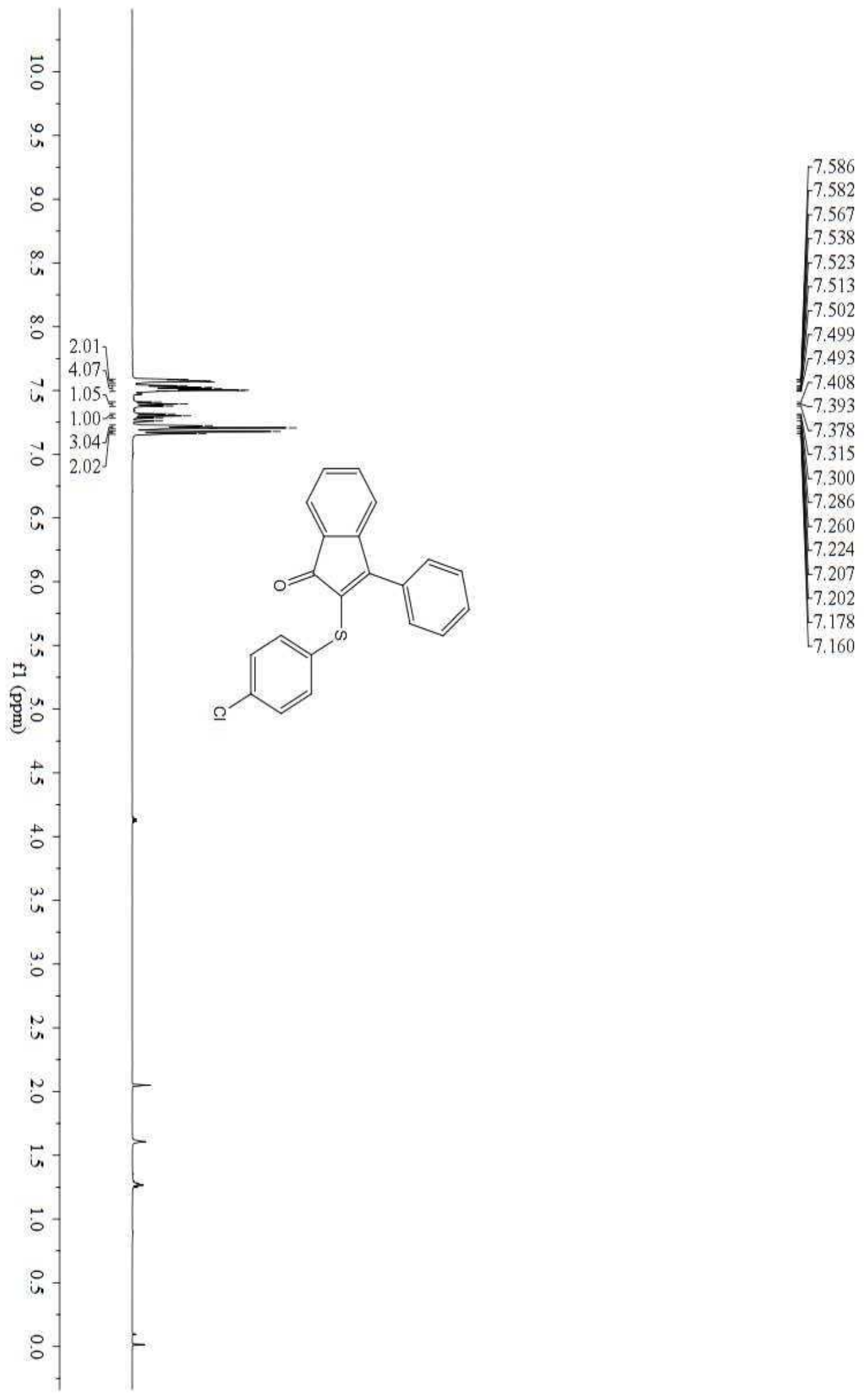


${ }^{13}$ C NMR of 2-((4-chlorophenyl)tho)-3-phenyl-1H-inden-1-one (11)

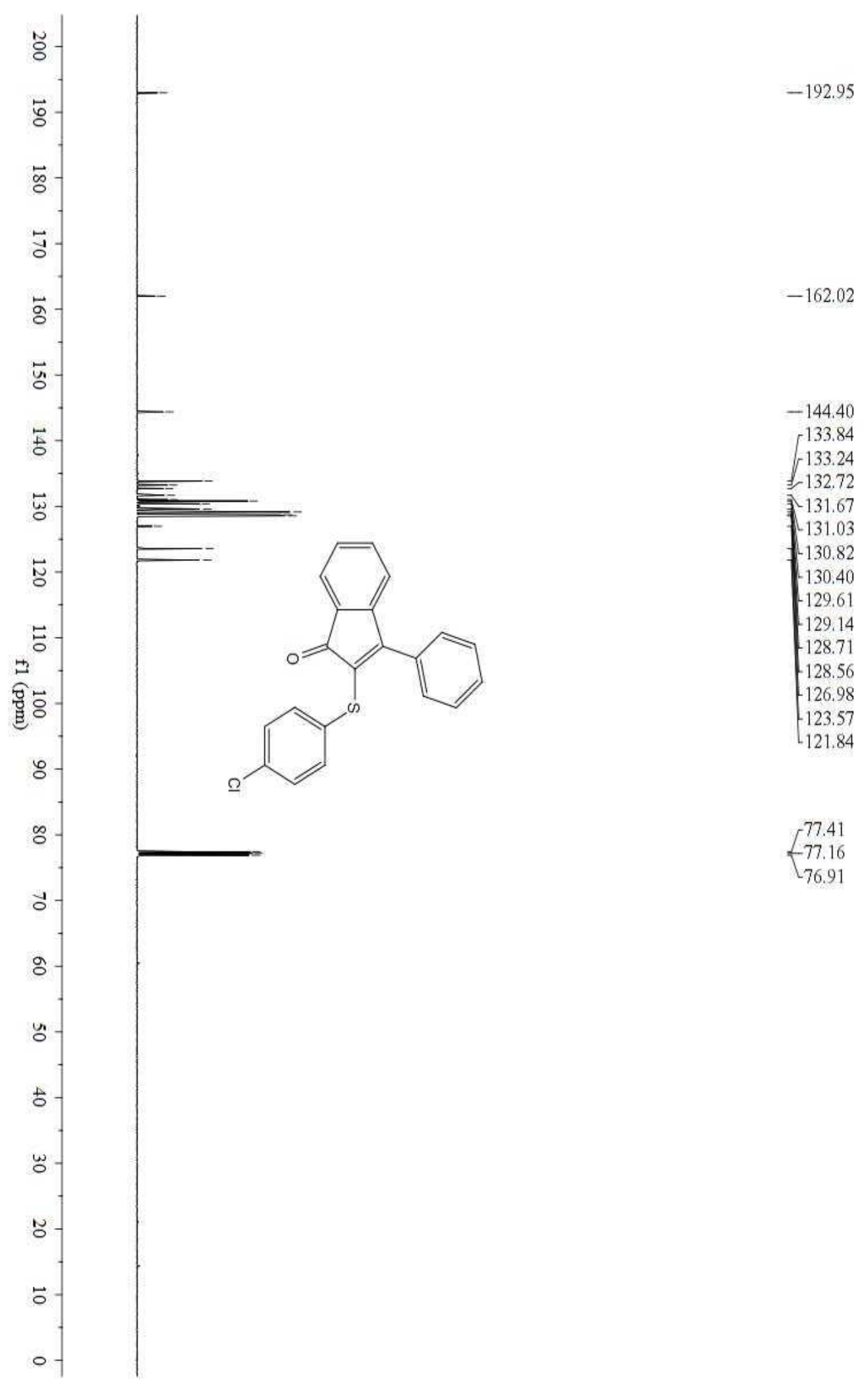




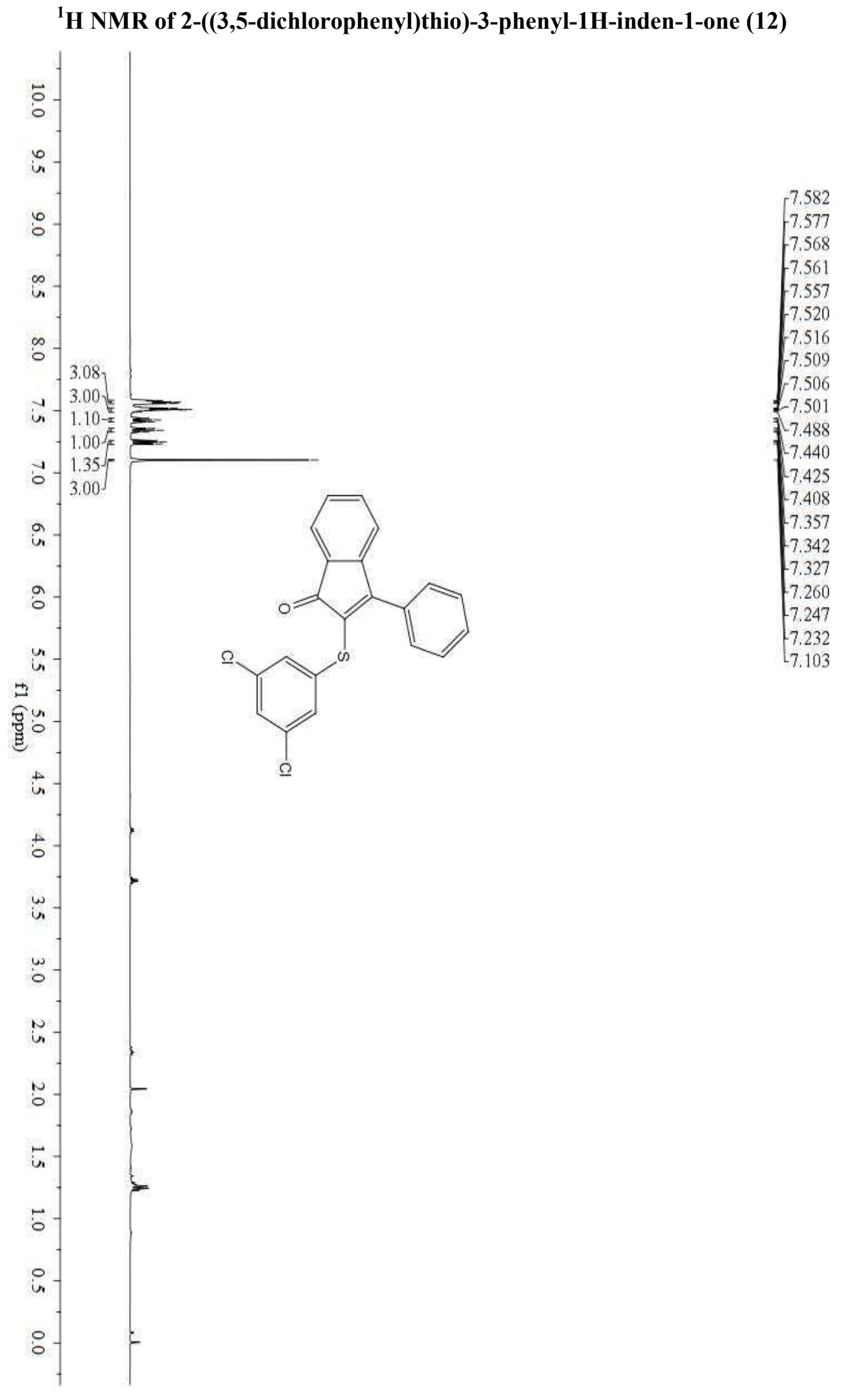


${ }^{13}$ C NMR of 2-((3,5-dichlorophenyl)thio)-3-phenyl-1H-inden-1-one (12)

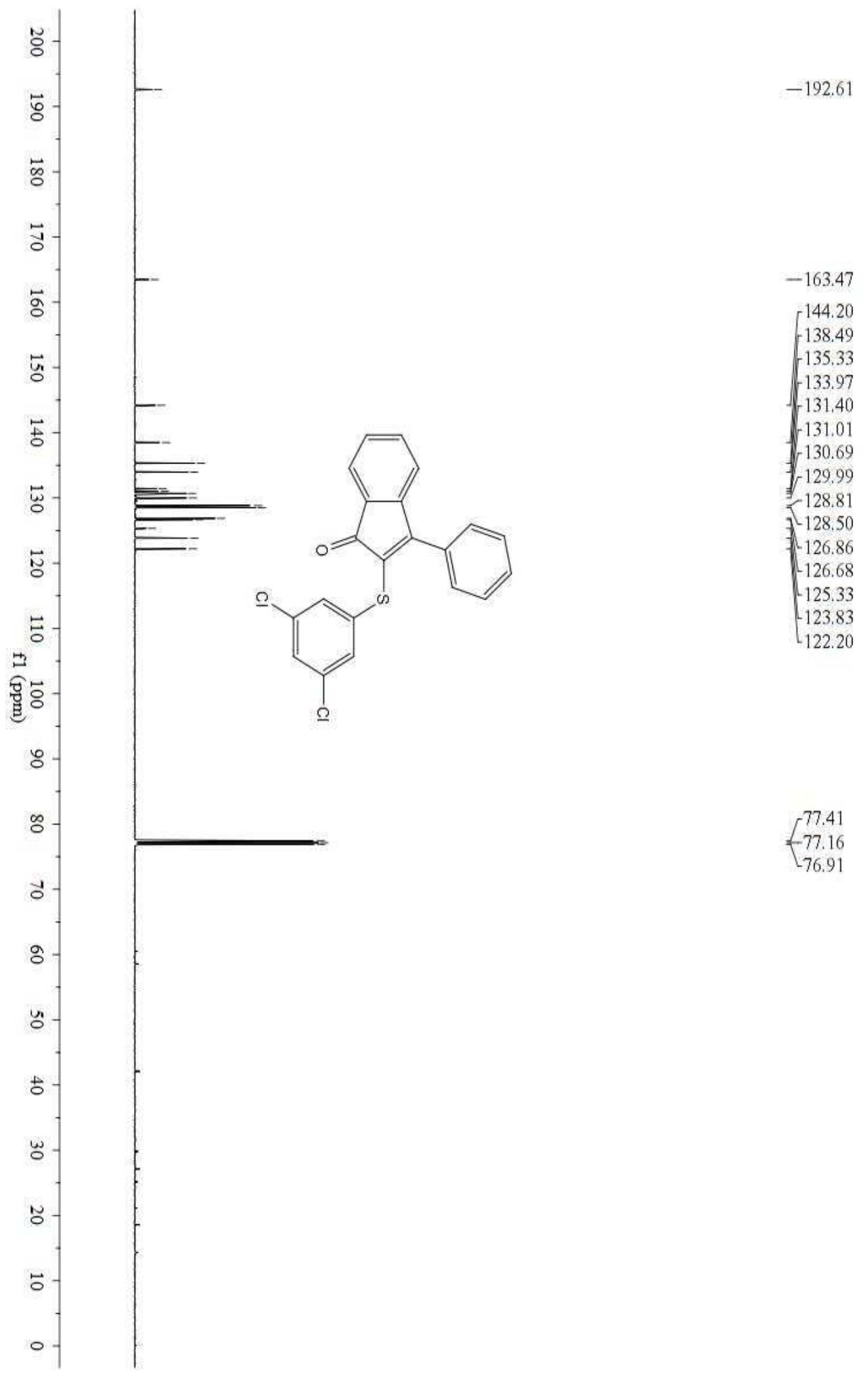




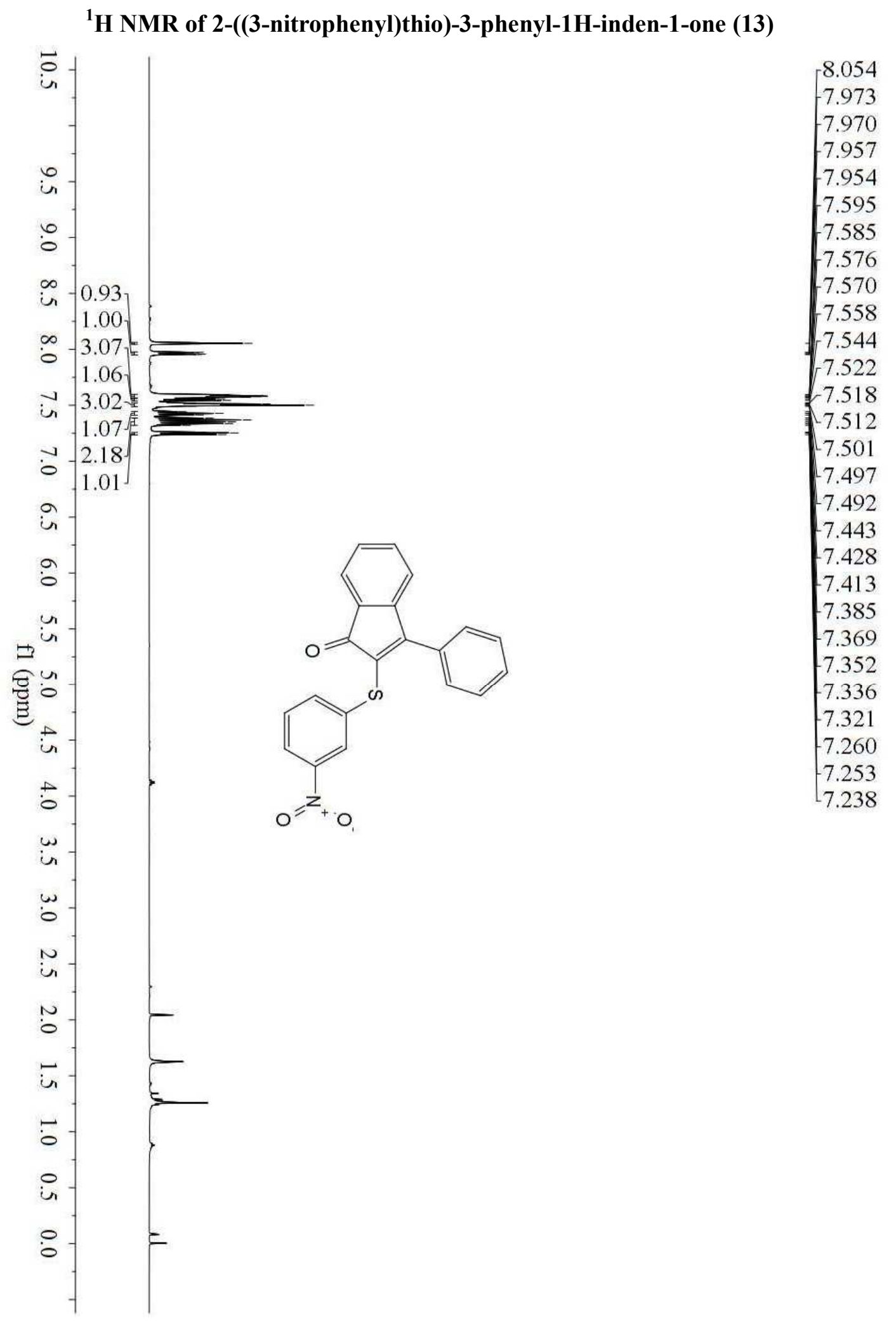




\section{${ }^{13}$ C NMR of 2-((3-nitrophenyl)thi)-3-phenyl-1H-inden-1-one (13)}

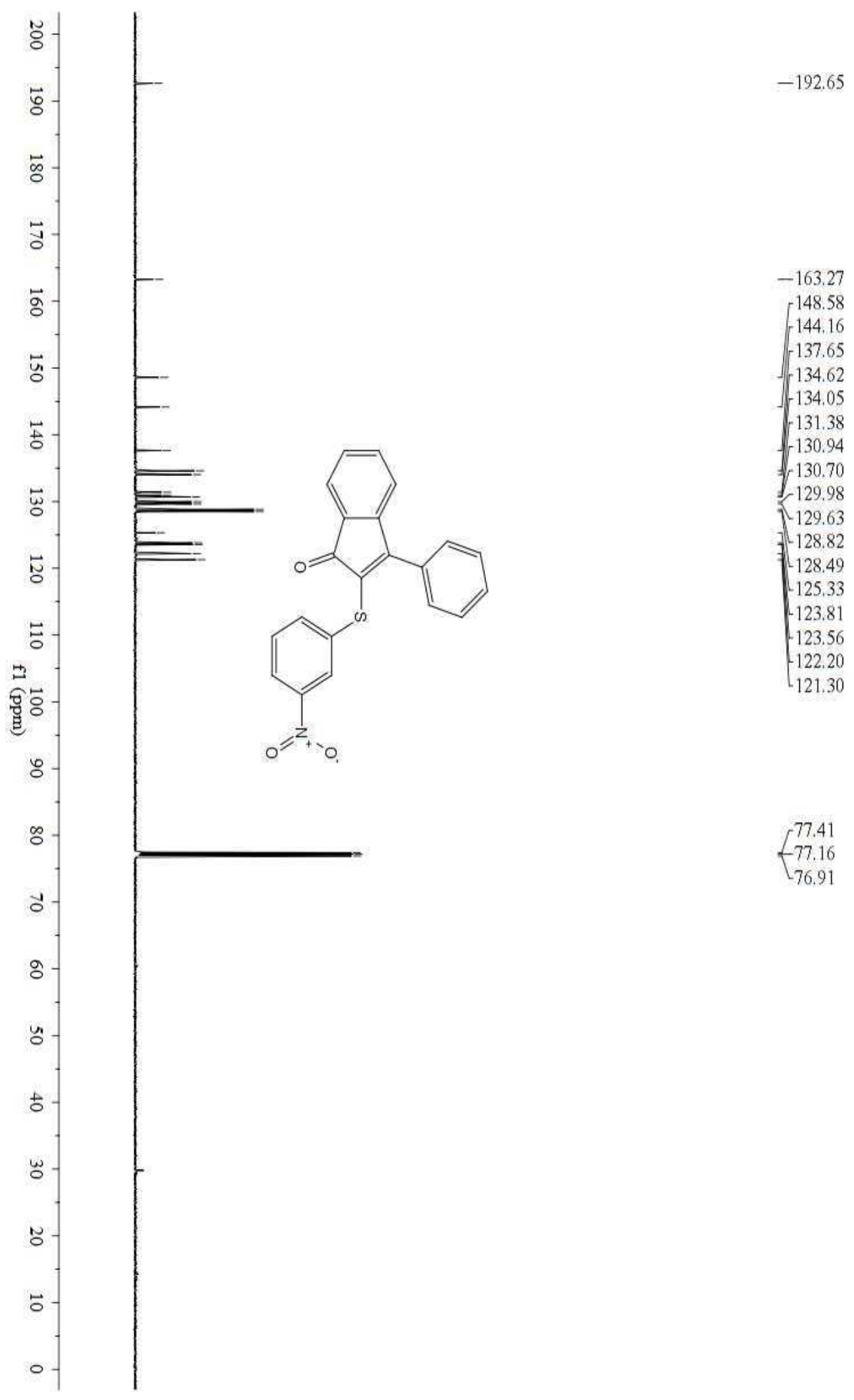




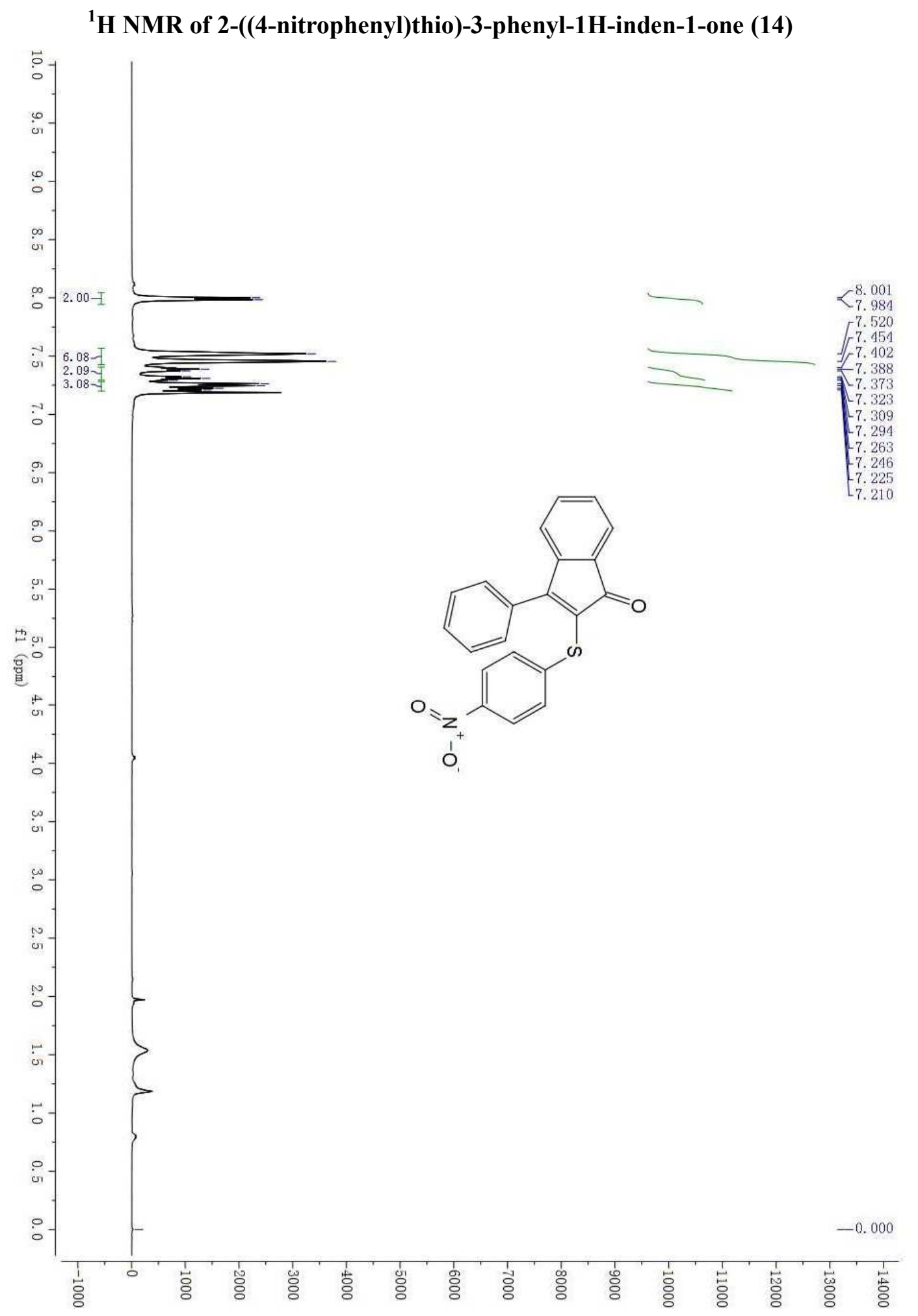


${ }^{13}$ C NMR of 2-((4-nitrophenyl)thio)-3-phenyl-1H-inden-1-one (14)

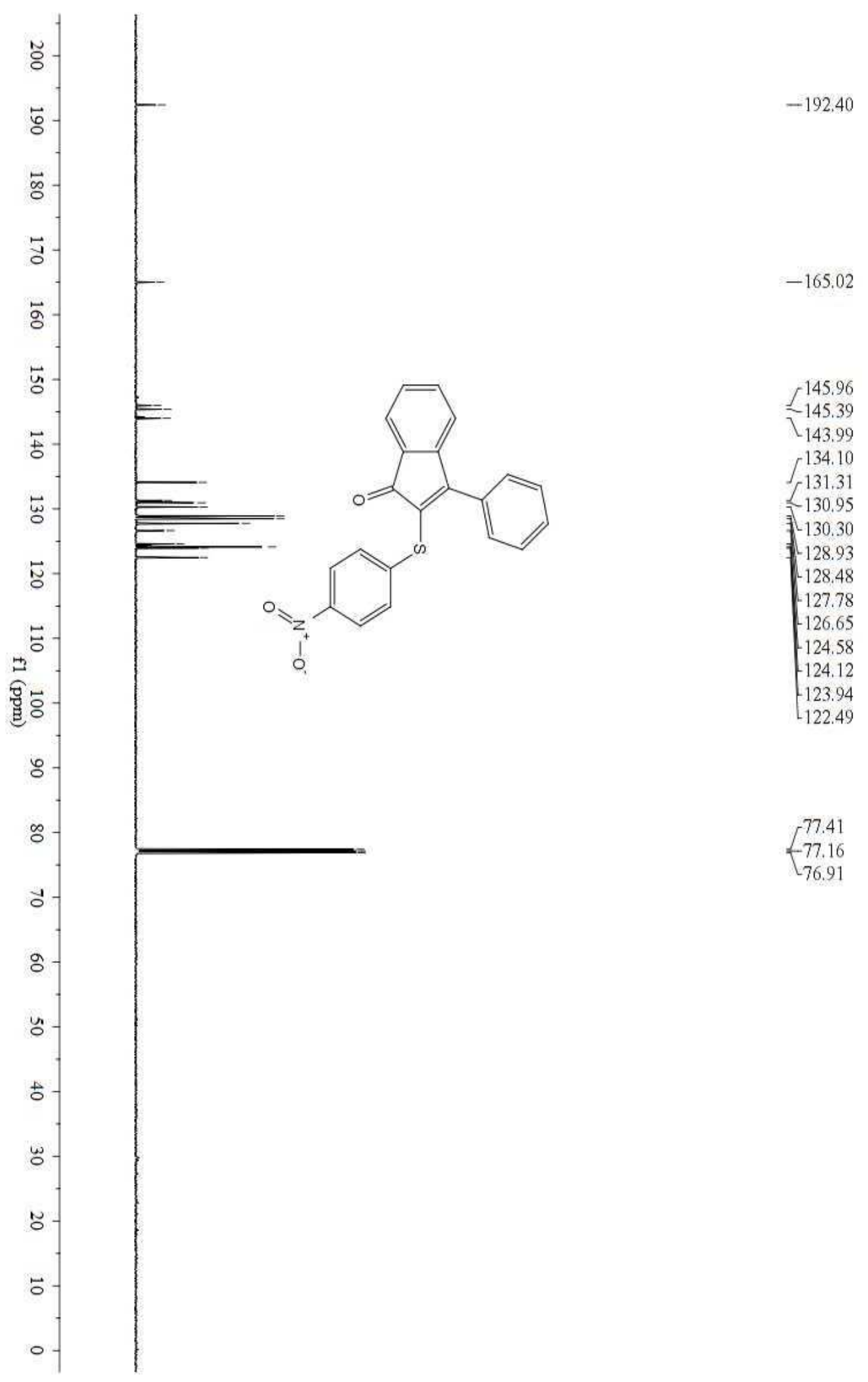


${ }^{1}$ H NMR of 2-(ethylthio)-3-phenyl-1H-inden-1-one (15)

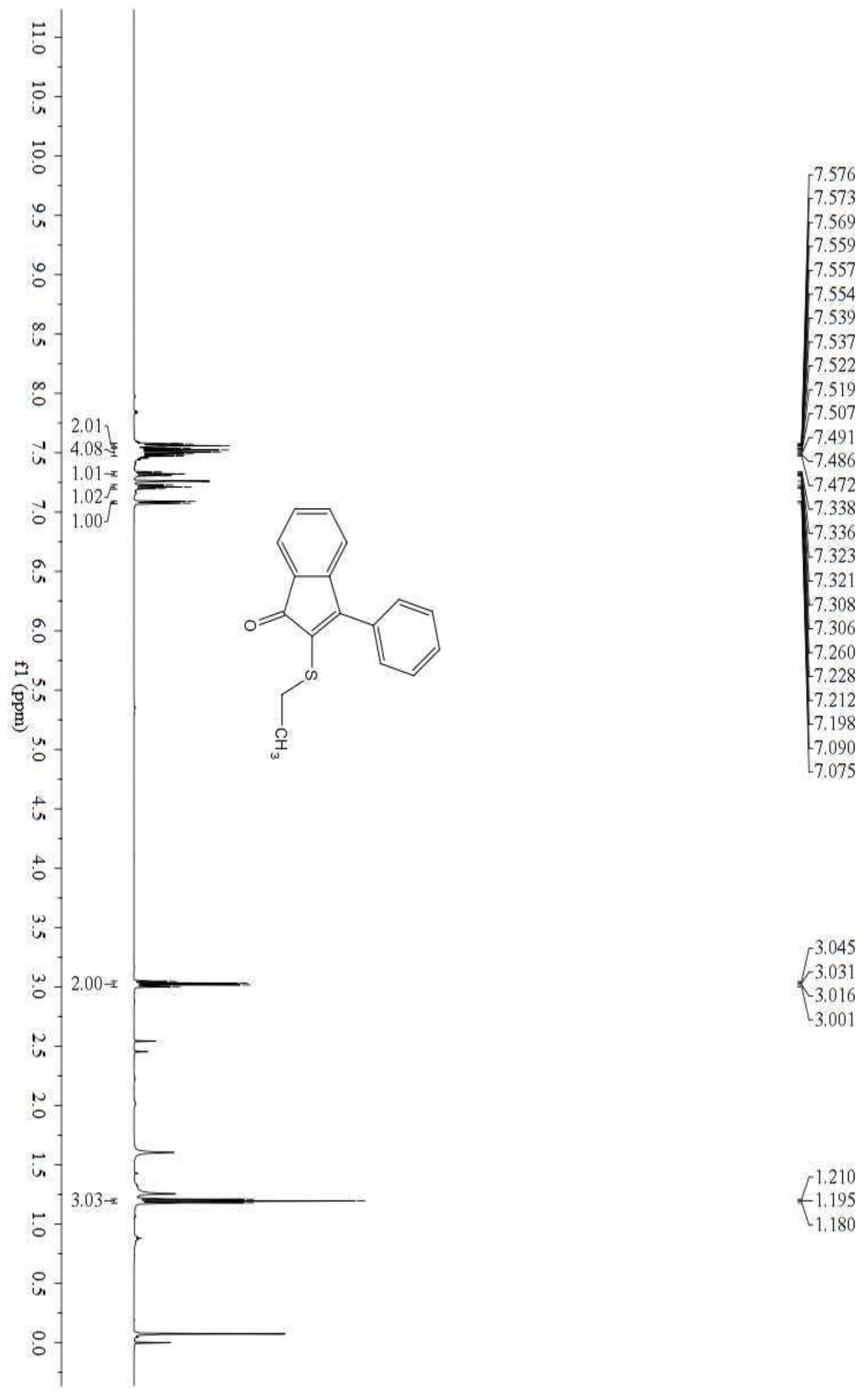




\section{${ }^{13}$ C NMR of 2-(ethylthio)-3-phenyl-1H-inden-1-one (15)}

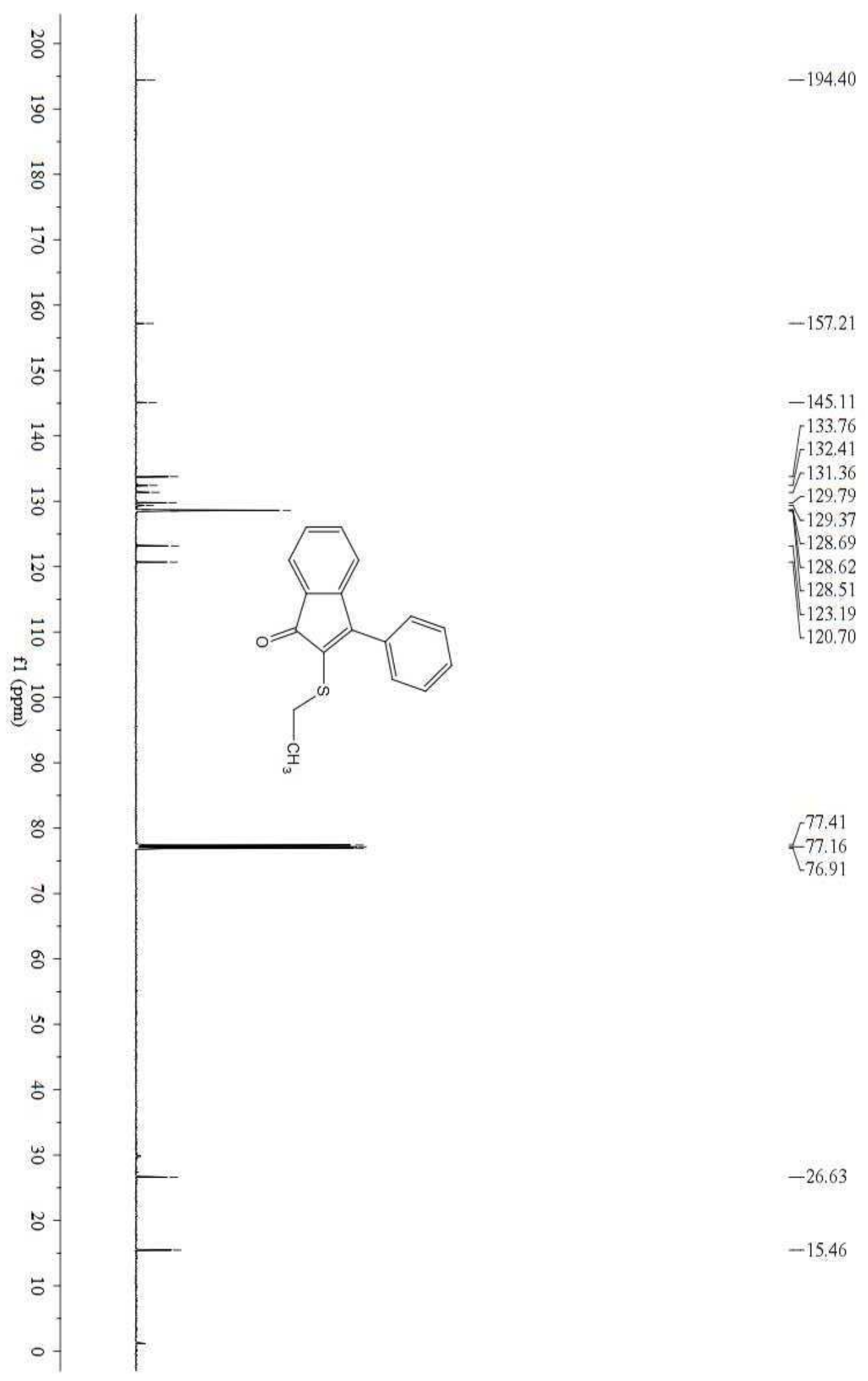


${ }^{1}$ H NMR of 2-(phenylthio)-3-(p-tolyl)-1H-inden-1-one (16)

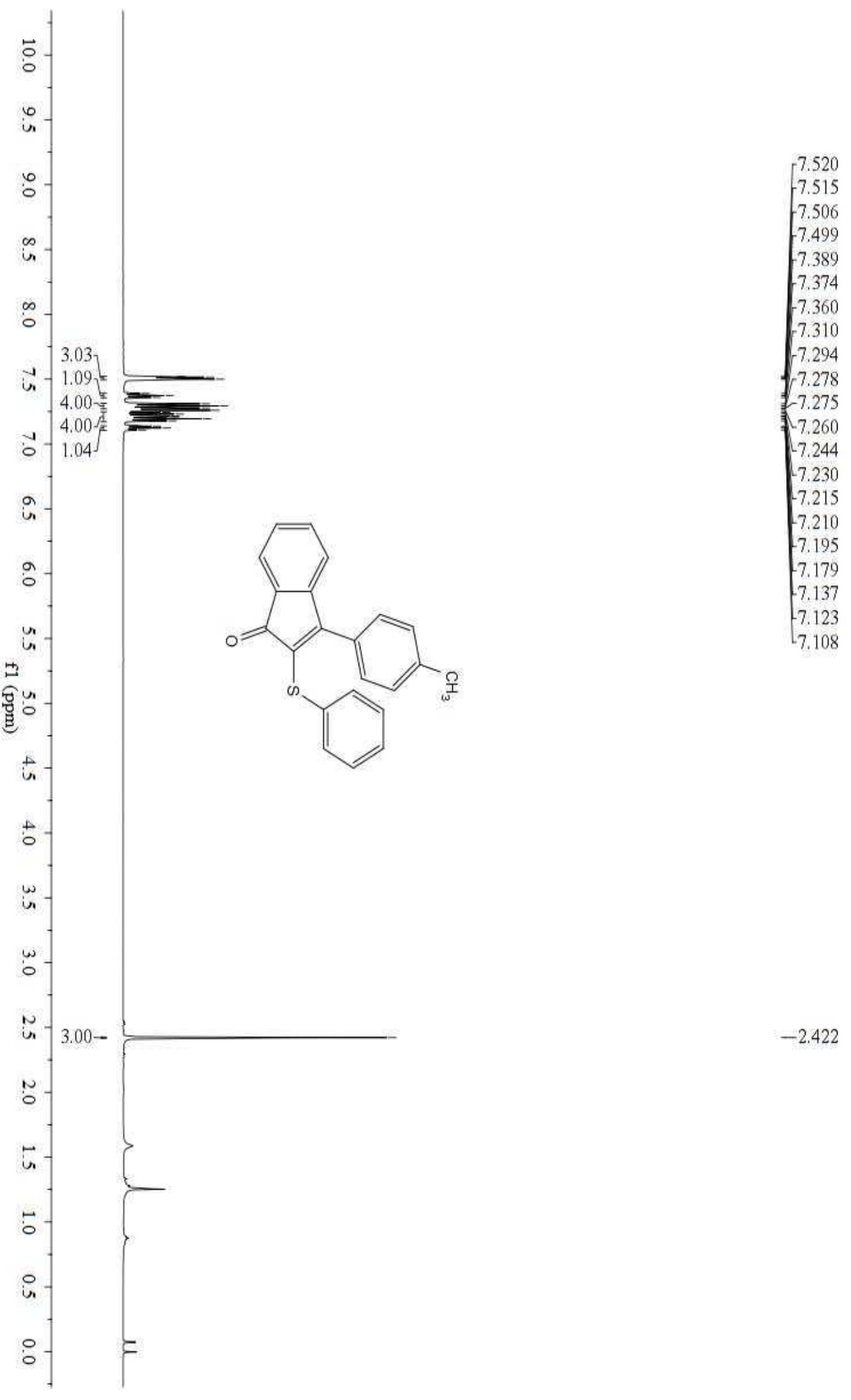




\section{${ }^{13}$ C NMR of 2-(phenylthio)-3-(p-tolyl)-1H-inden-1-one (16)}

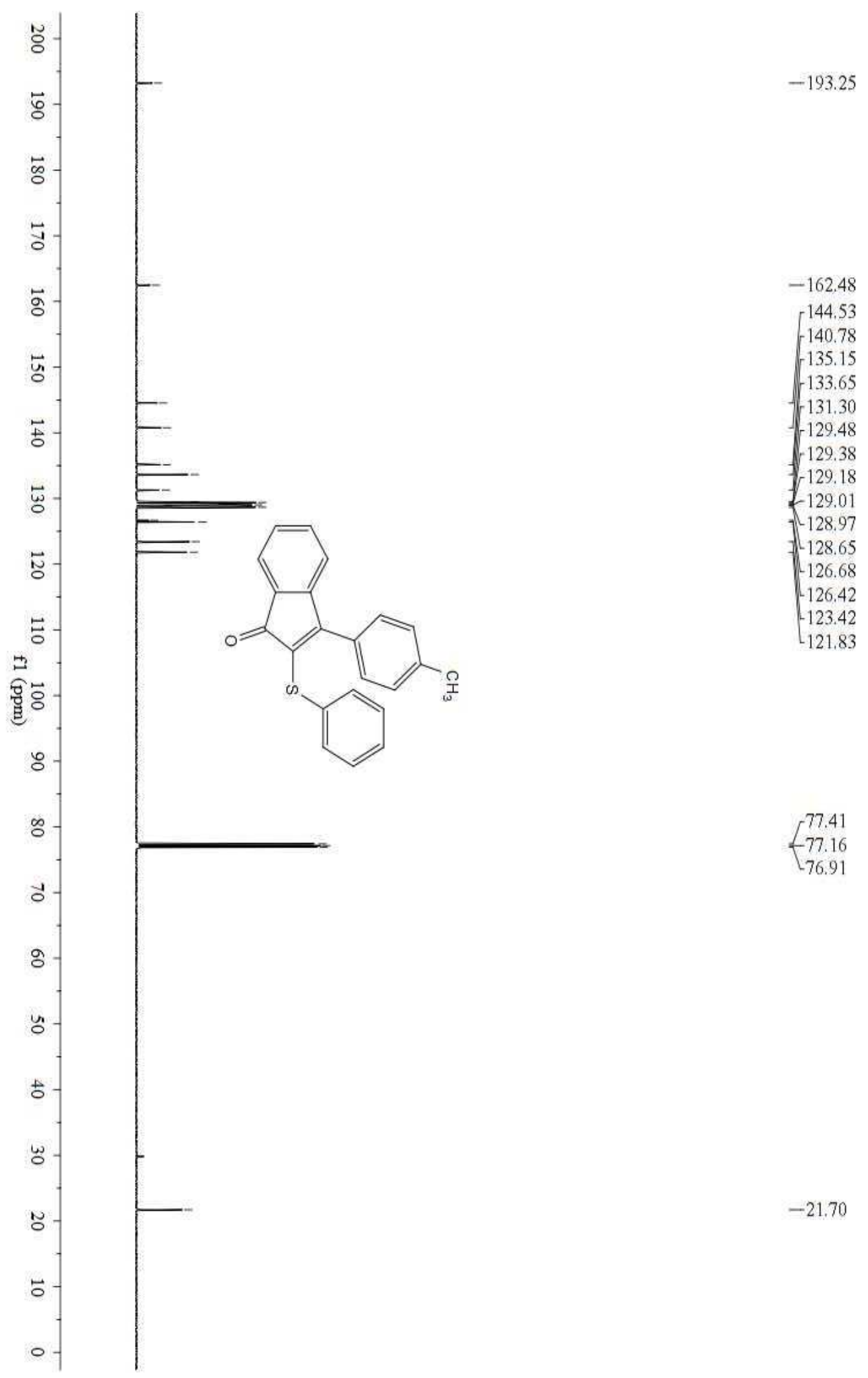


${ }^{1}$ H NMR of 2-(phenylthio)-3-(o-tolyl)-1H-inden-1-one (17)

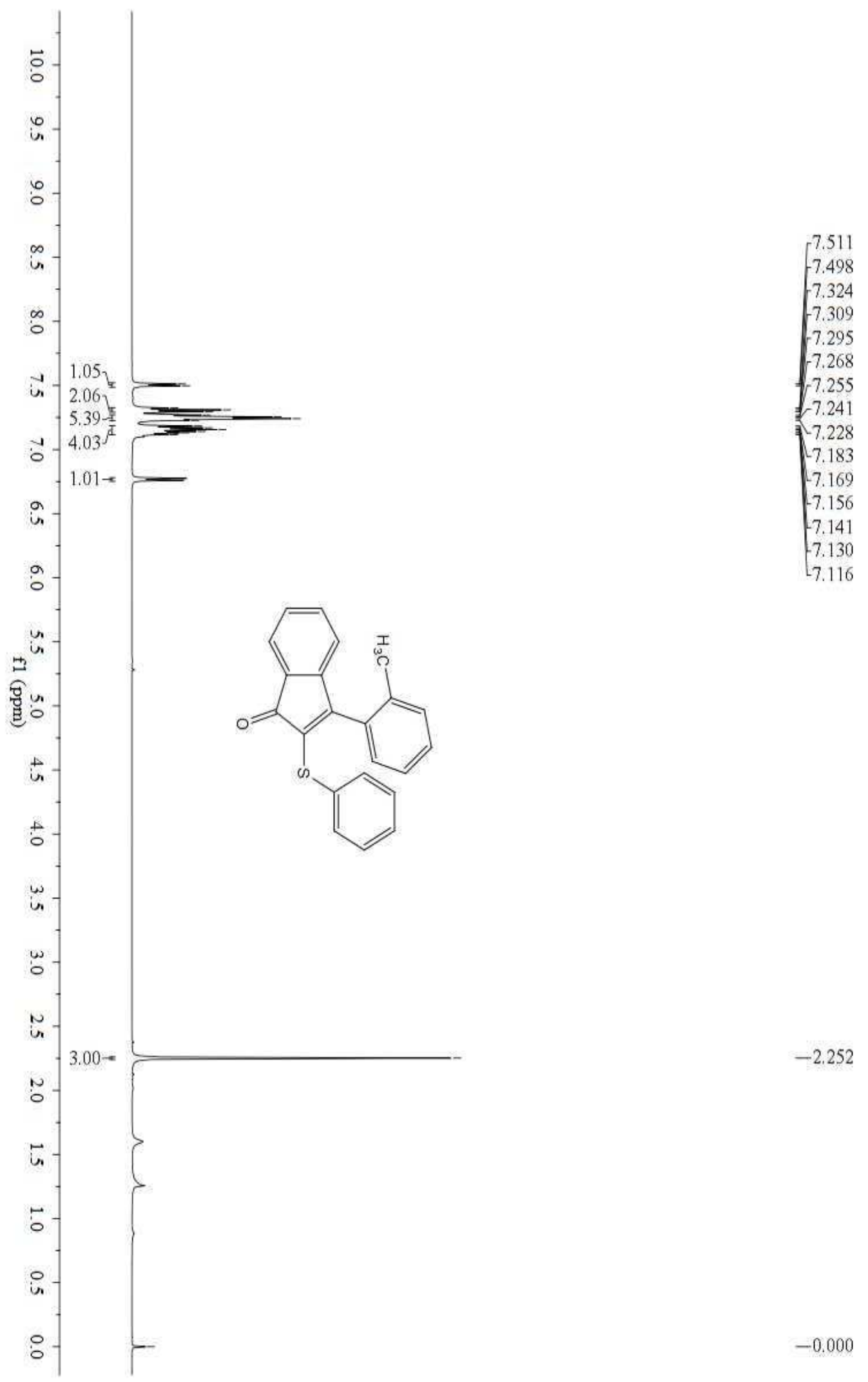




\section{${ }^{13}$ C NMR of 2-(phenylthio)-3-(o-tolyl)-1H-inden-1-one (17)}

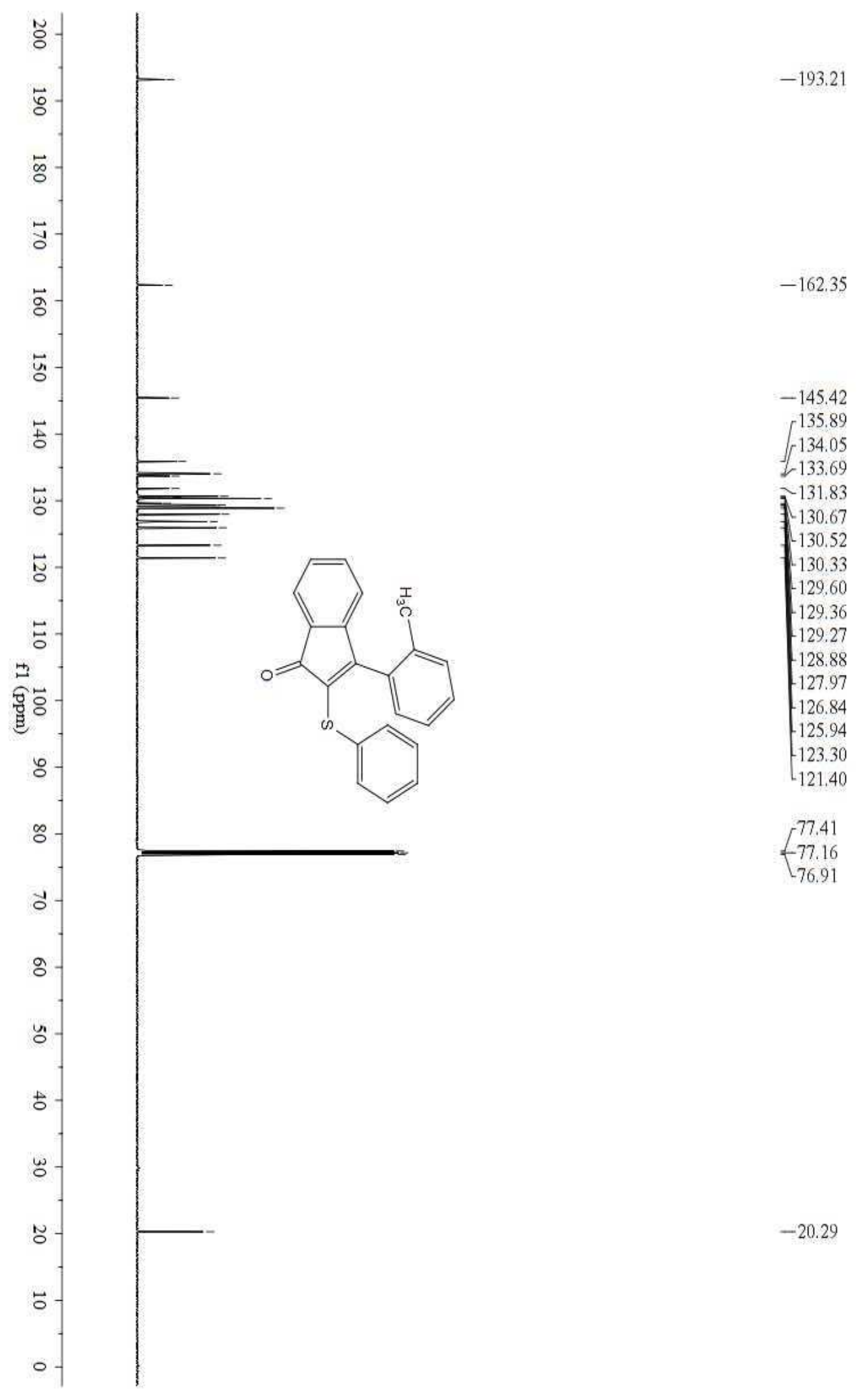


${ }^{1}$ H NMR of 3-(4-ethylphenyl)-2-(phenylthio)-1H-inden-1-one (18)

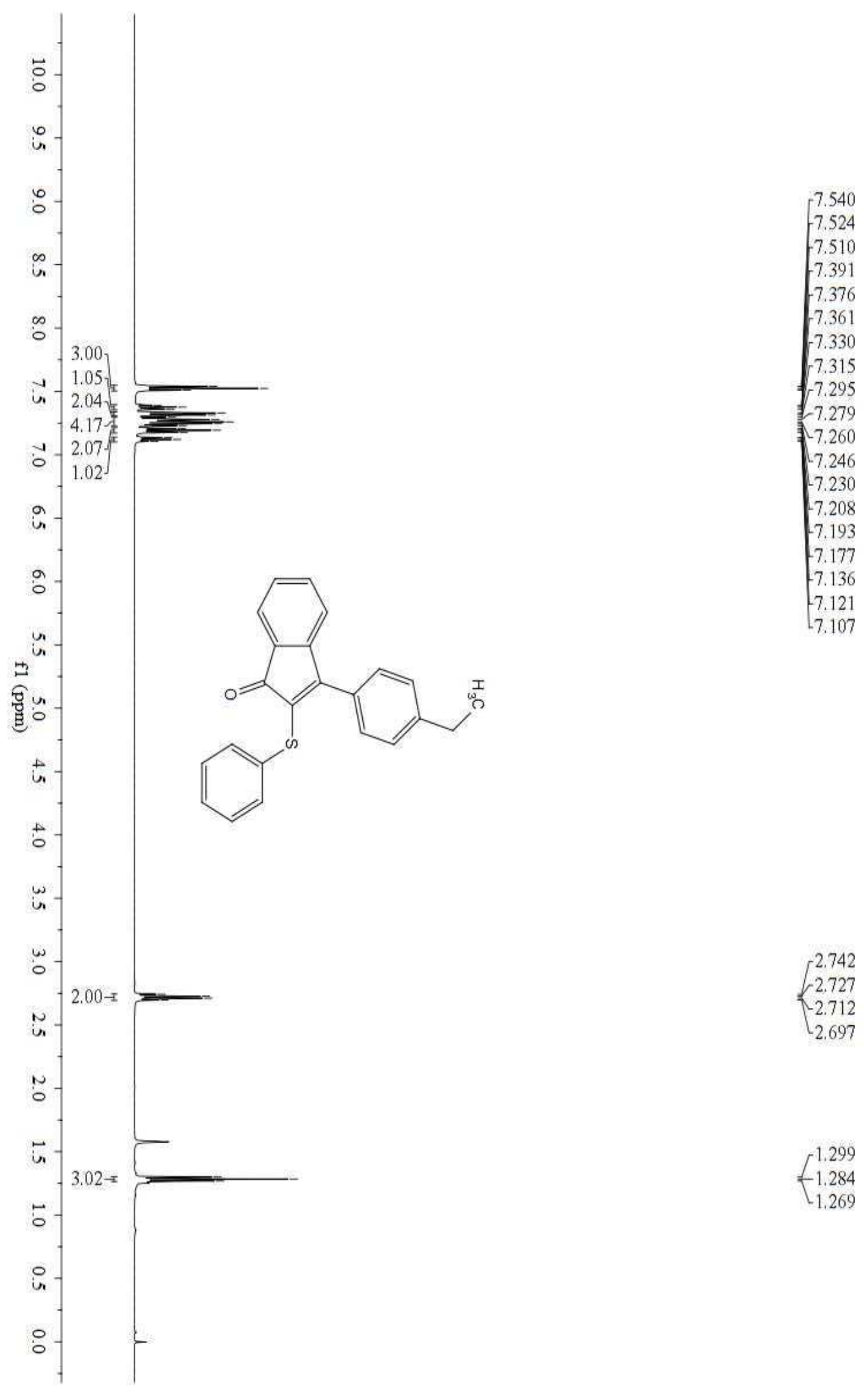


${ }^{13}$ C NMR of 3-(4-ethylphenyl)-2-(phenylthio)-1H-inden-1-one (18)

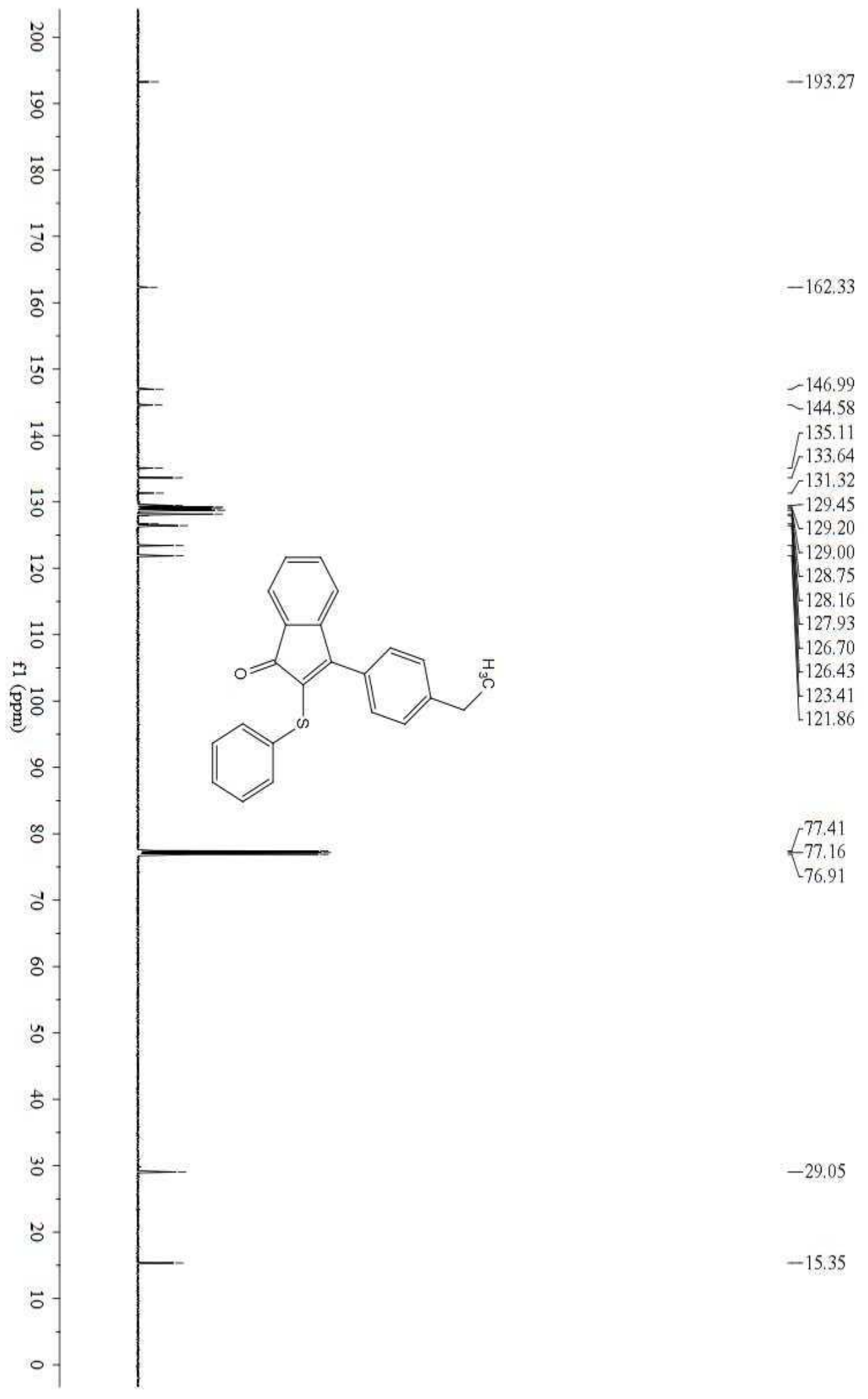


${ }^{1}$ H NMR of 3-(2-fluorophenyl)-2-(phenylthio)-1 H-inden-1-one (19)

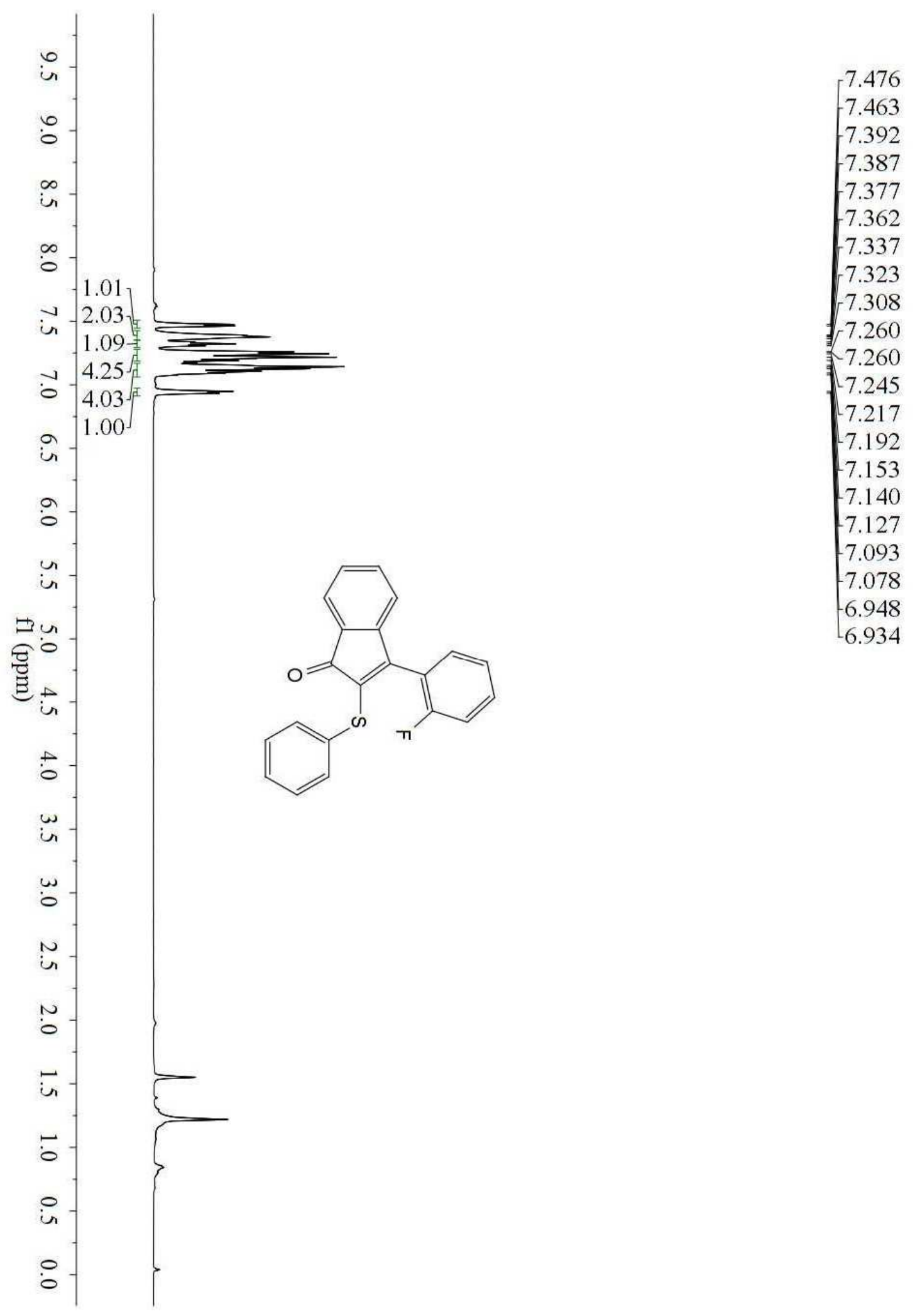




\section{${ }^{13}$ C NMR of 3-(2-fluorophenyl)-2-(phenylthio)-1H-inden-1-one (19)}

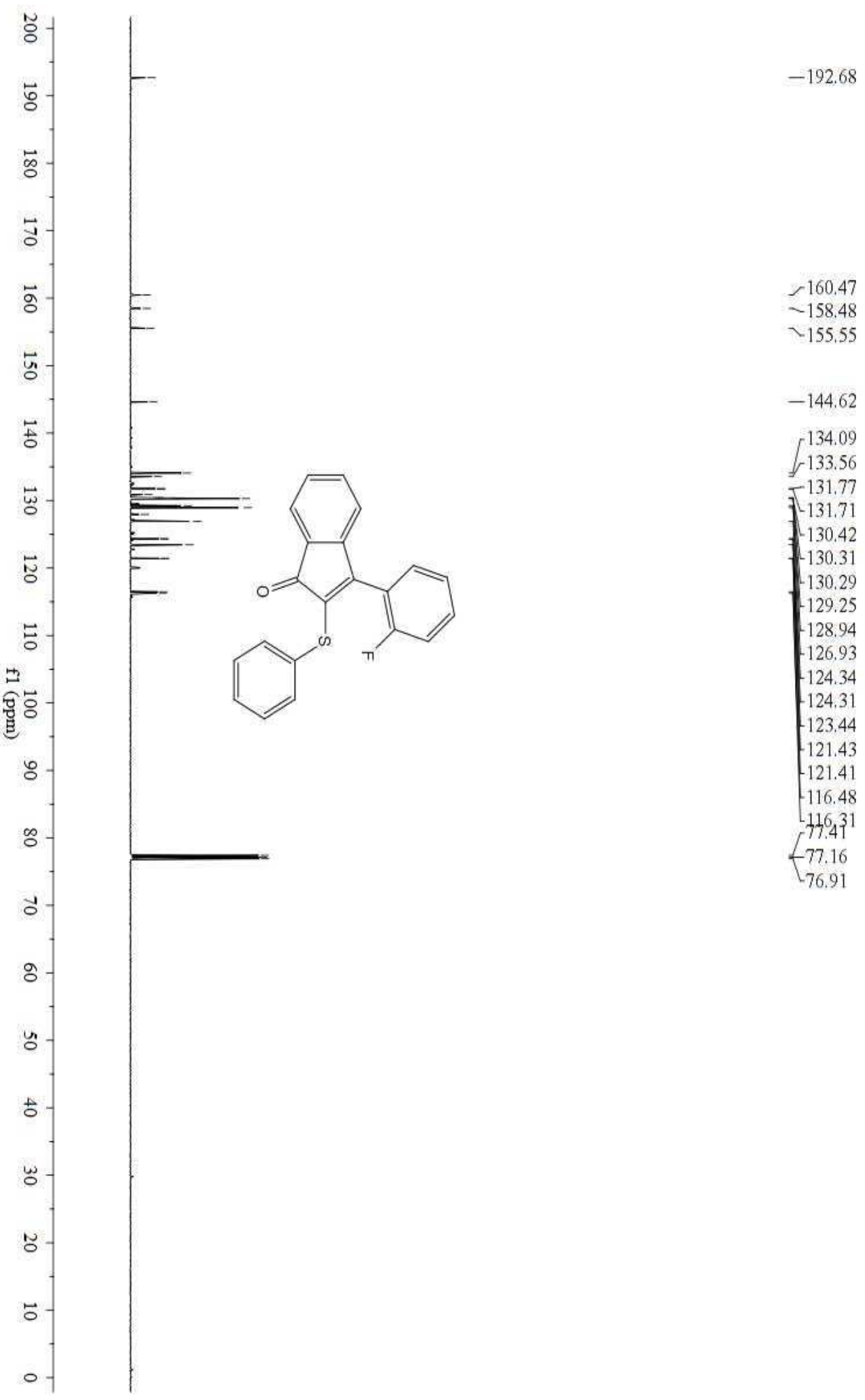




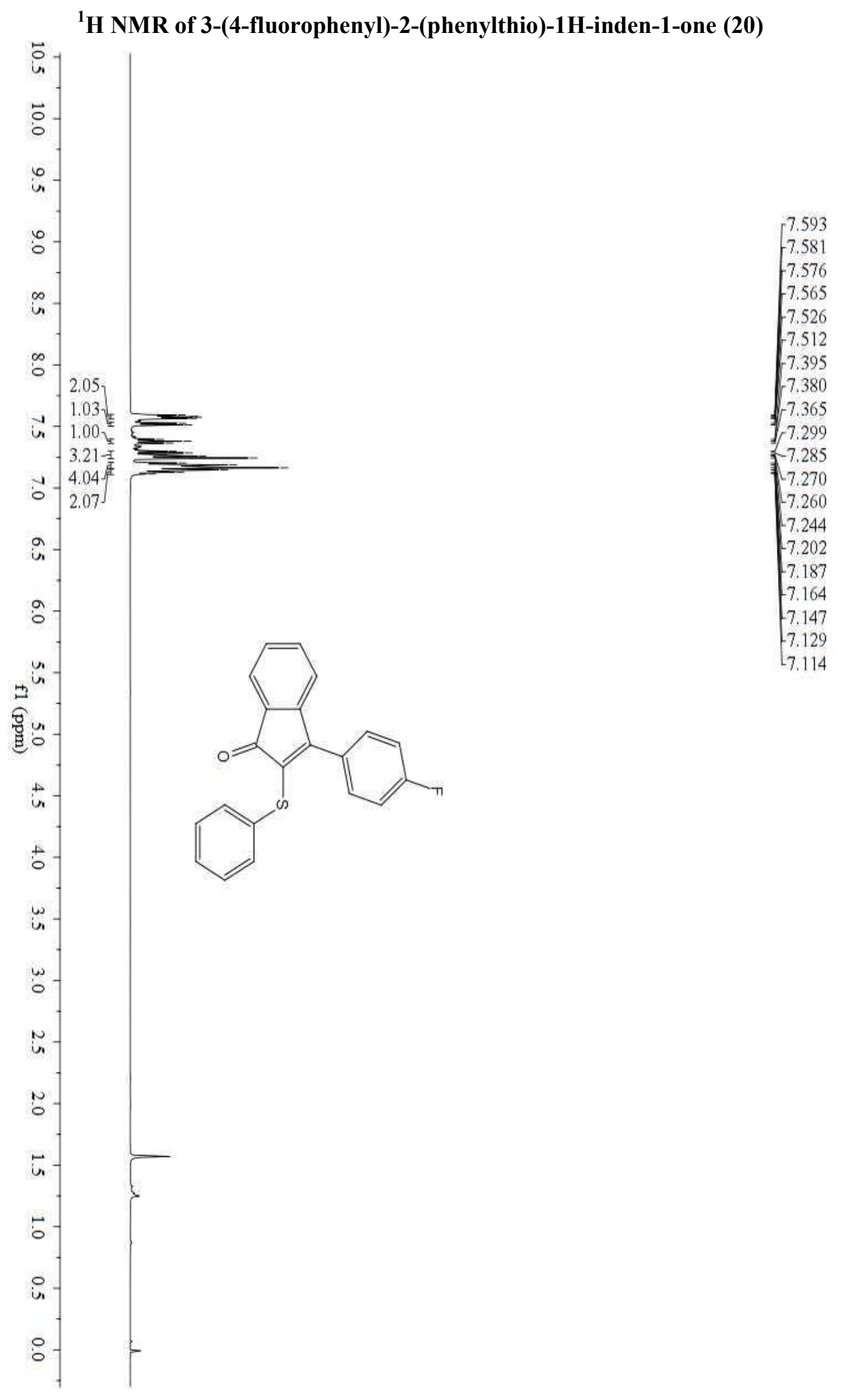


${ }^{13}$ C NMR of 3-(4-fluorophenyl)-2-(phenylthio)-1H-inden-1-one (20)

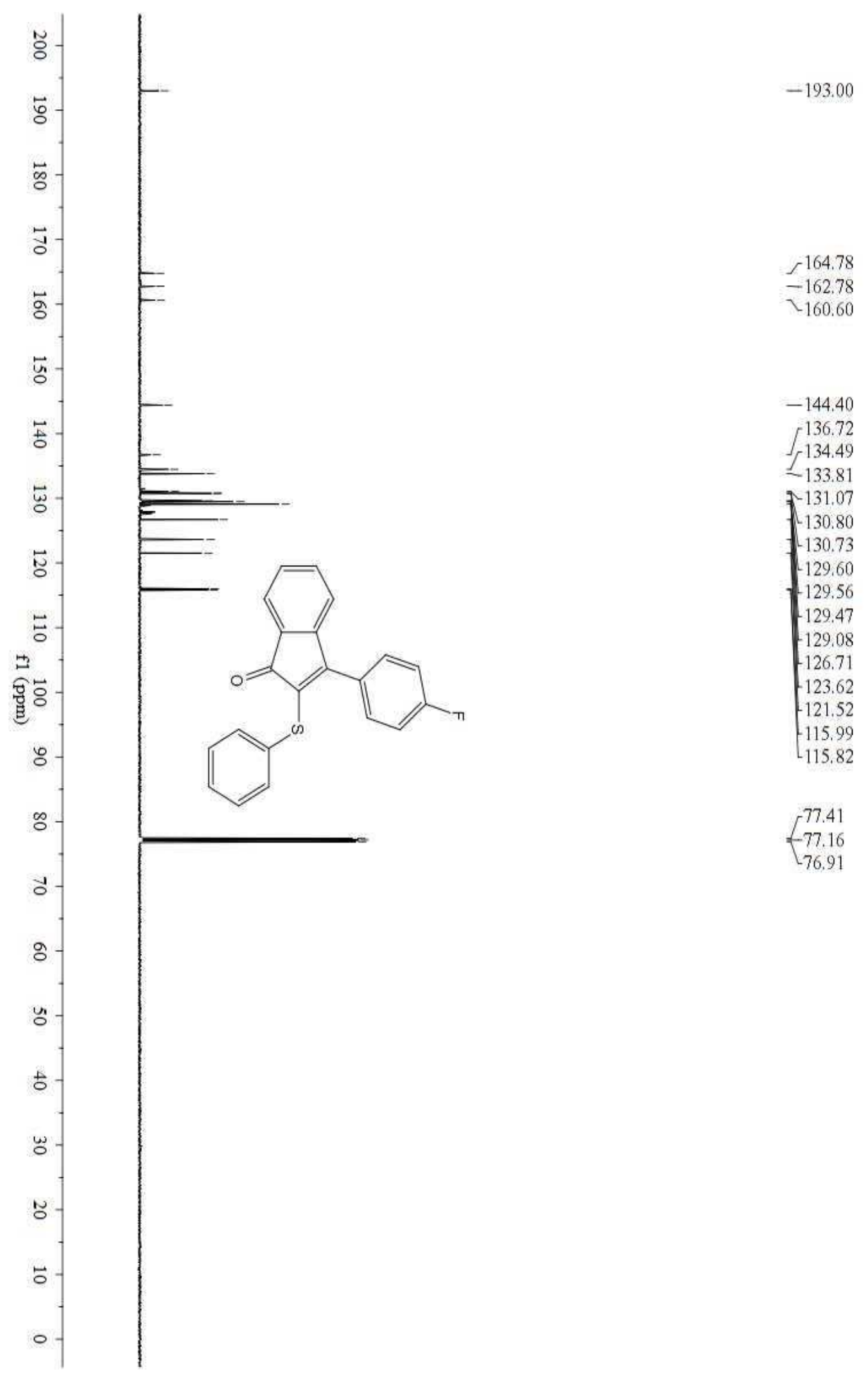


${ }^{1}$ H NMR of 3-(4-chlorophenyl)-2-(phenylthio)-1H-inden-1-one (21)

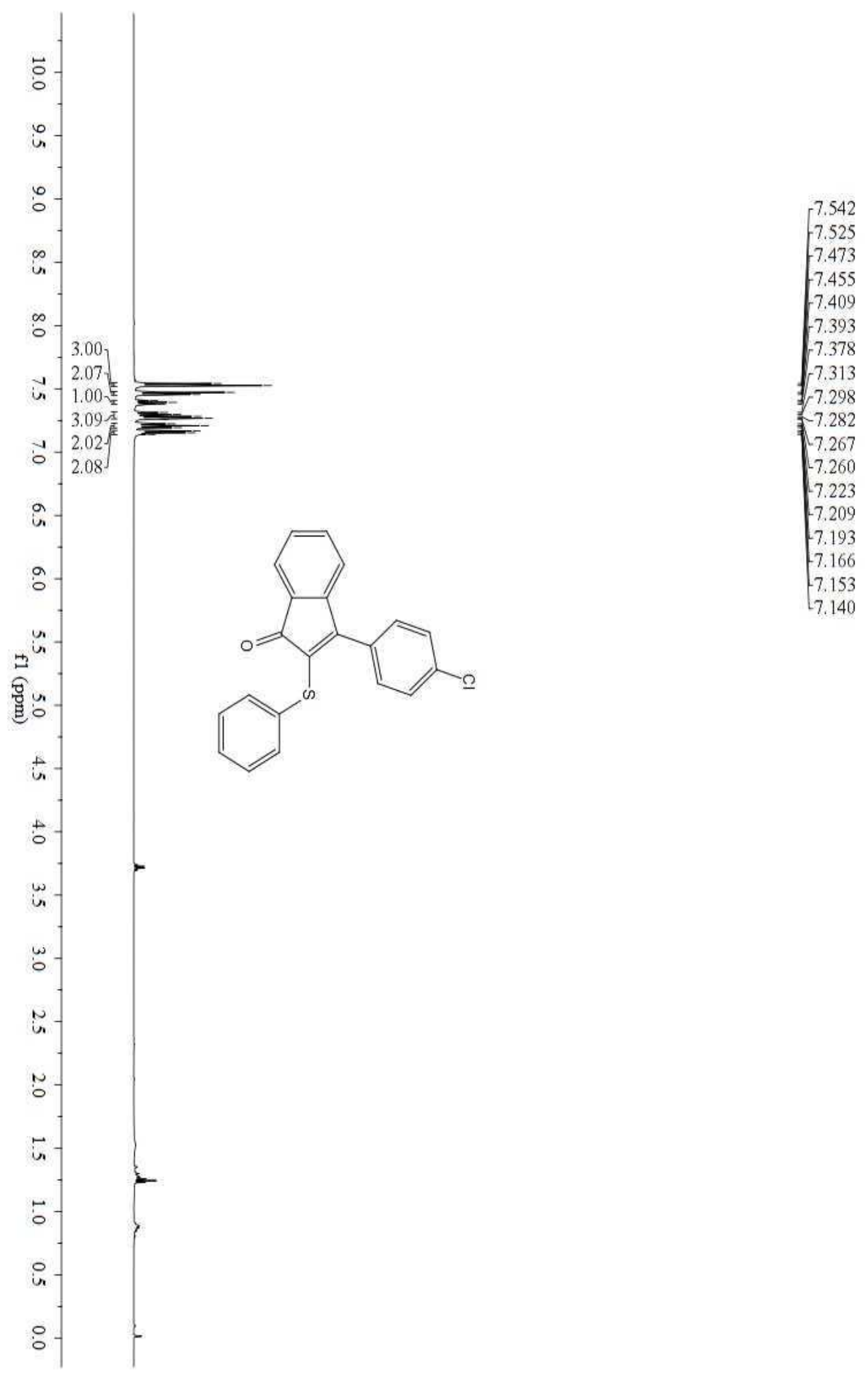


${ }^{13}$ C NMR of 3-(4-chlorophenyl)-2-(phenylthio)-1H-inden-1-one (21)

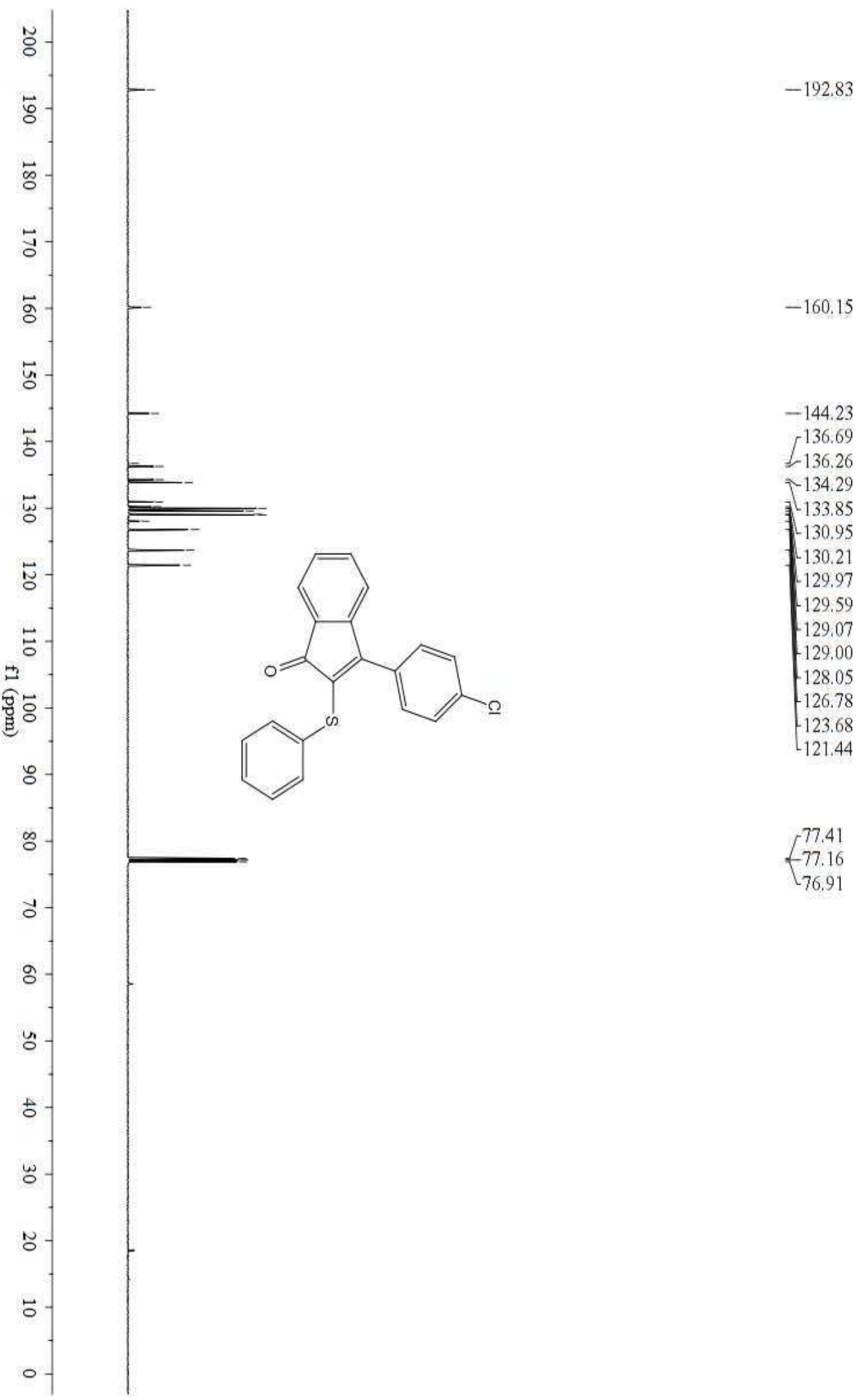


${ }^{1}$ H NMR of 3-cyclohexyl-2-(phenylthio)-1H-inden-1-one (22)

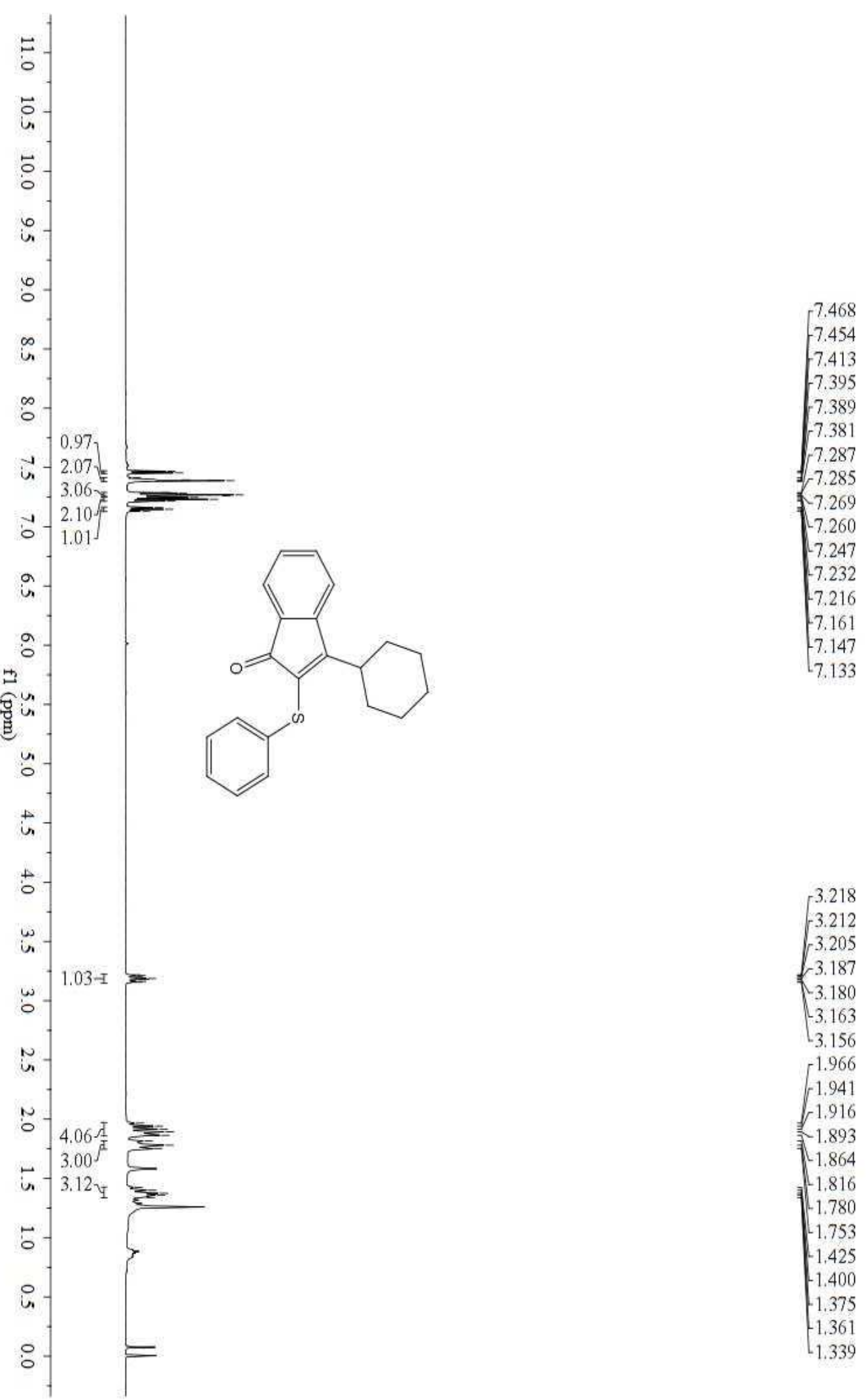




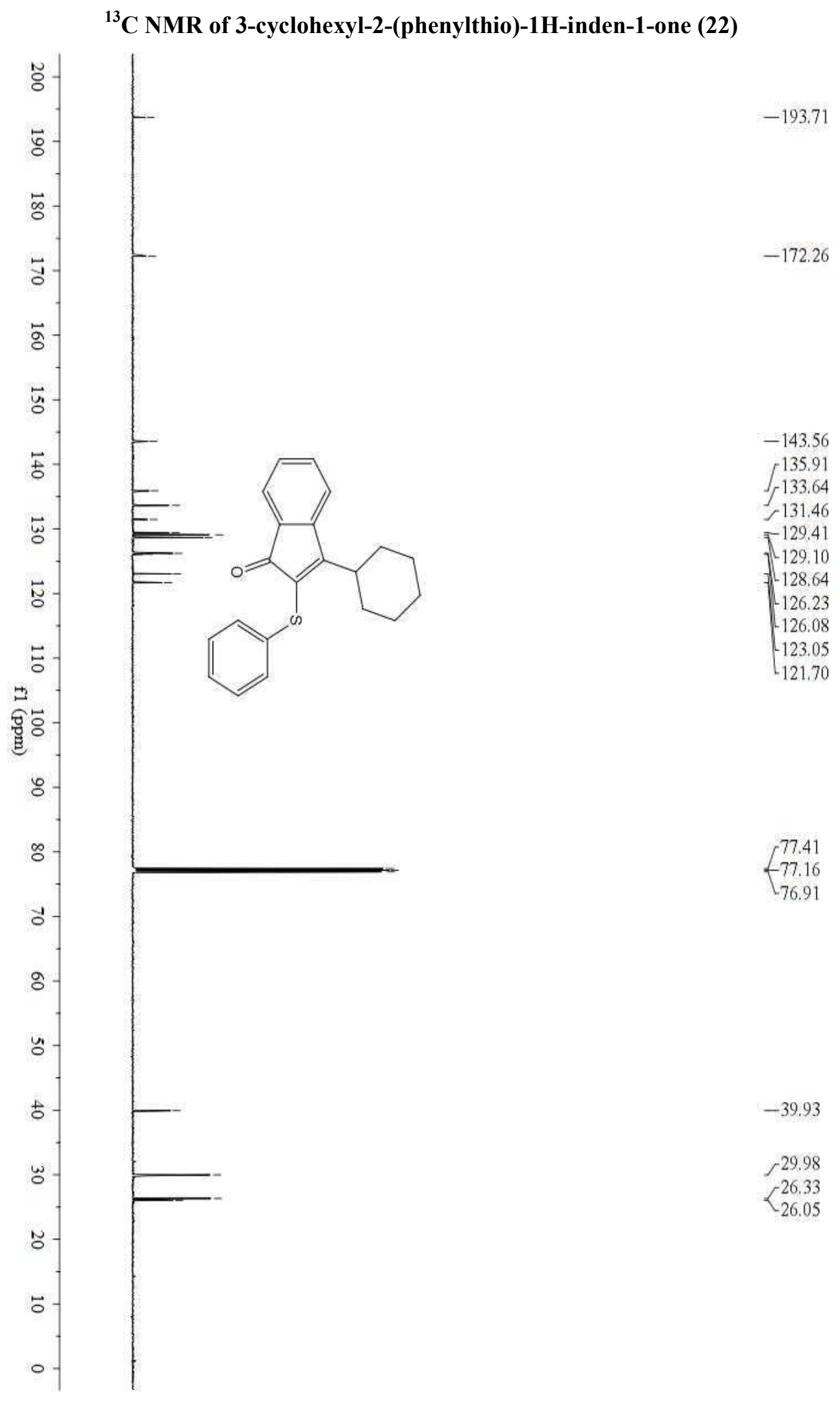


${ }^{1}$ H NMR of 5-methyl-3-phenyl-2-(phenylthio)-1H-inden-1-one (23)

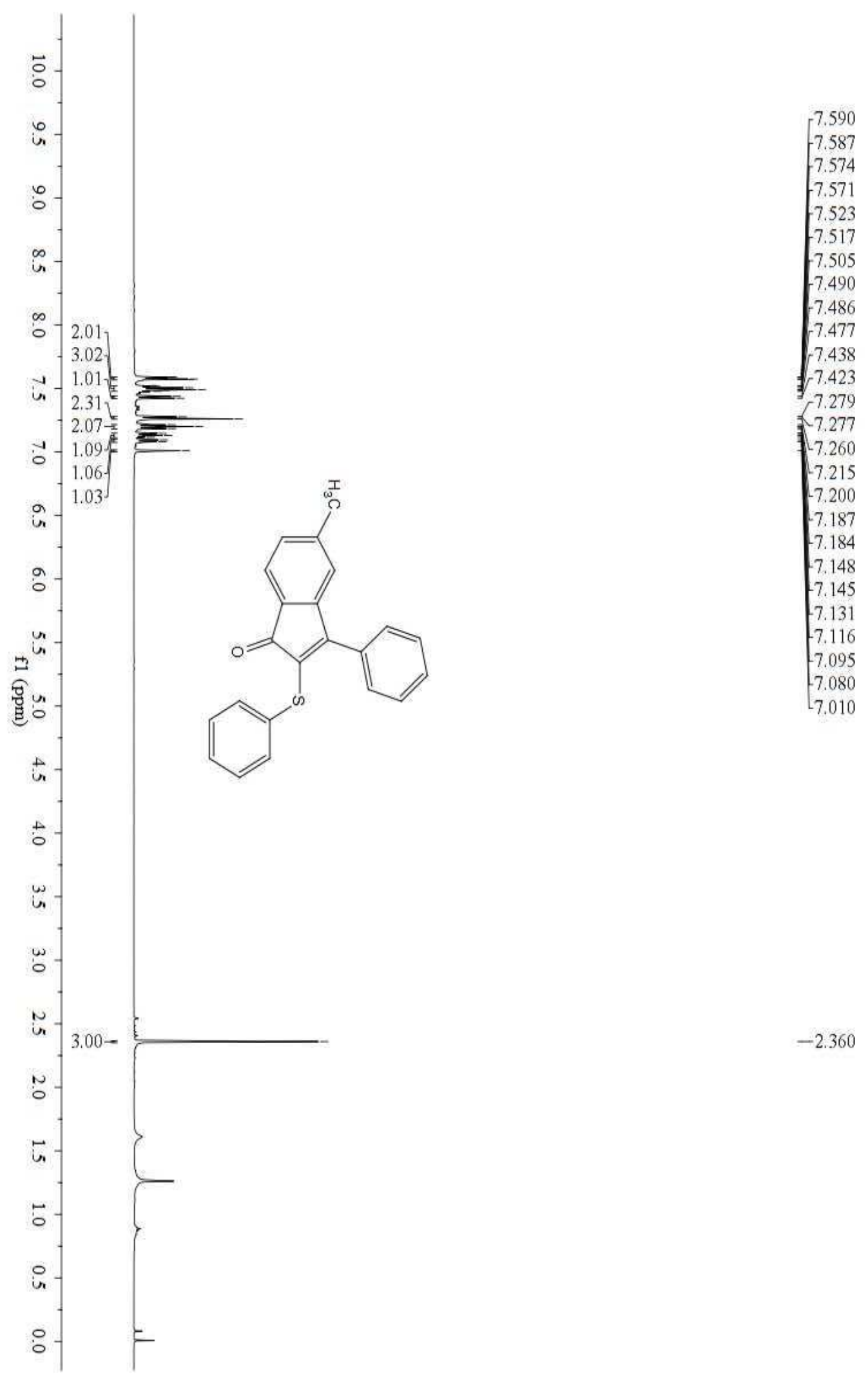


${ }^{13}$ C NMR of 5-methyl-3-phenyl-2-(phenylthio)-1H-inden-1-one (23)

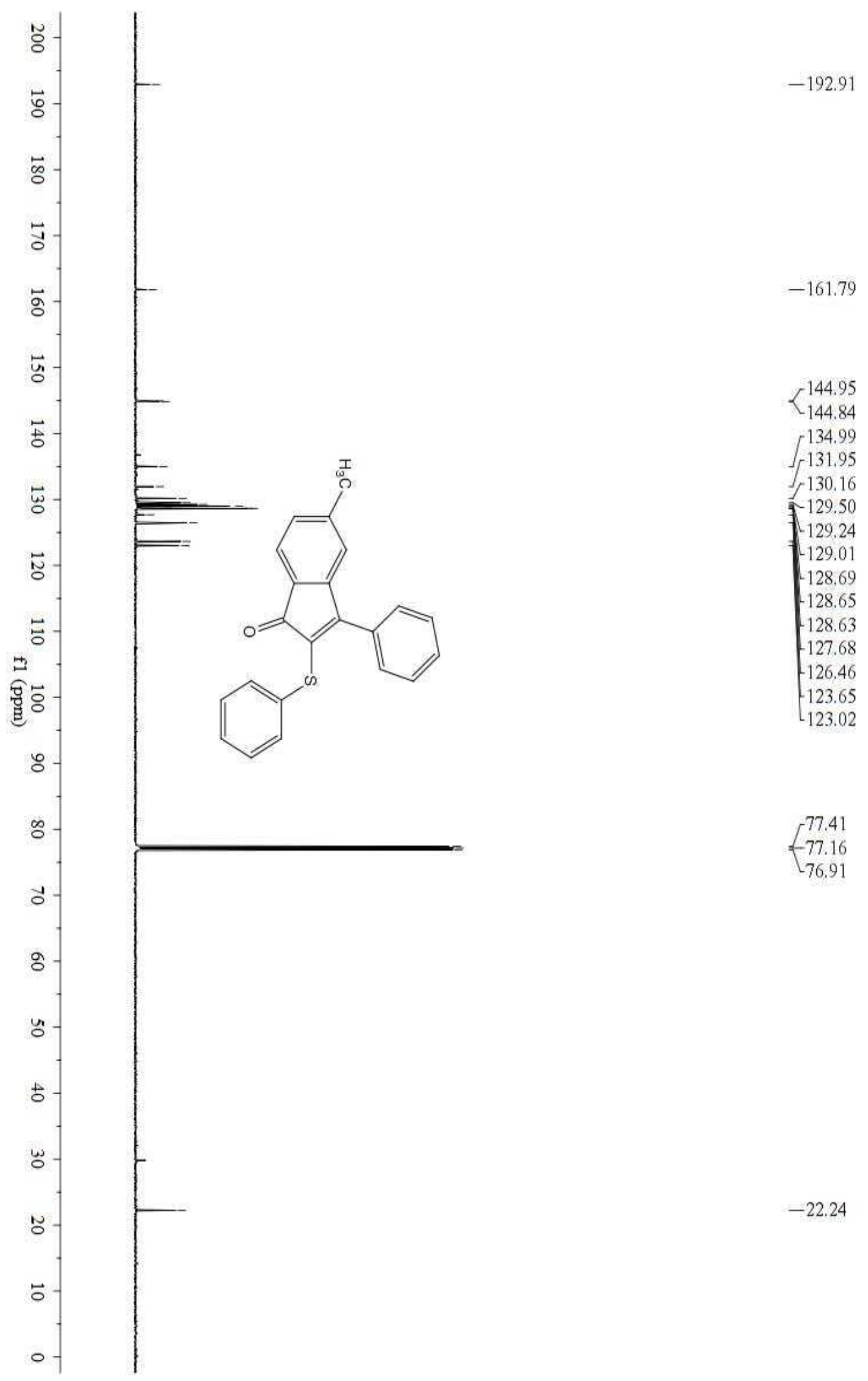




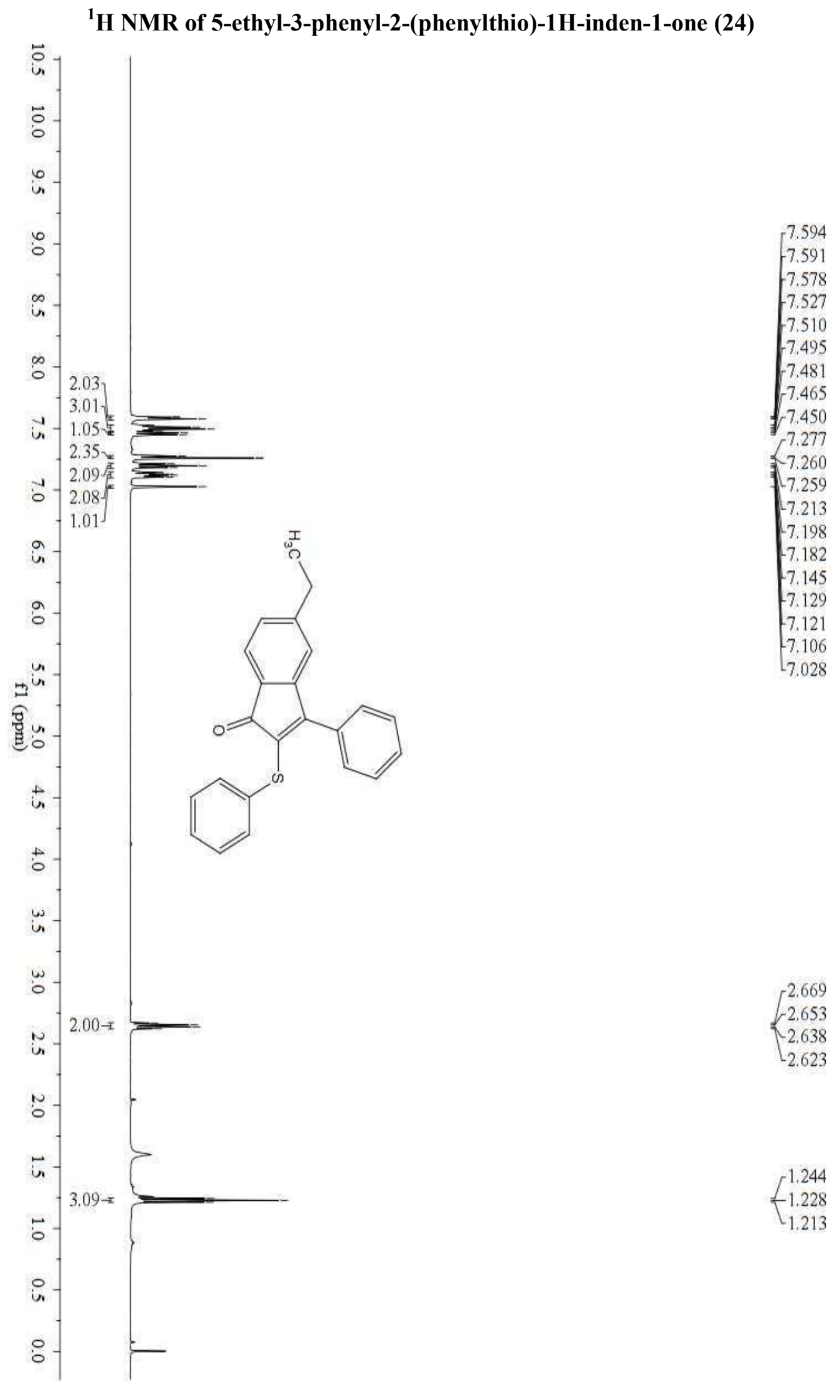


${ }^{13}$ C NMR of 5-ethyl-3-phenyl-2-(phenylthio)-1H-inden-1-one (24)

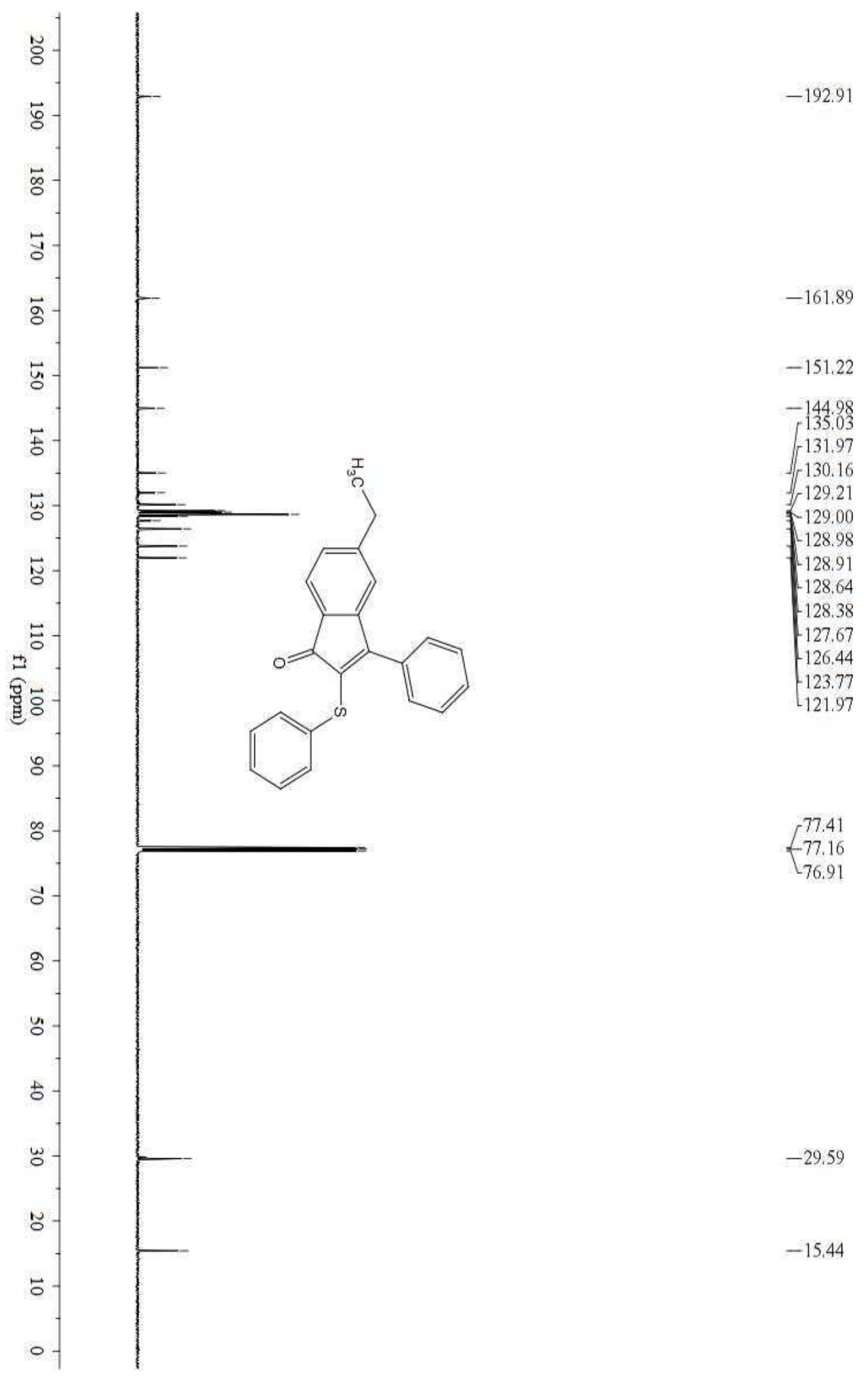


${ }^{1}$ H NMR of 5-butyl-3-phenyl-2-(phenylthio)-1H-inden-1-one (25)

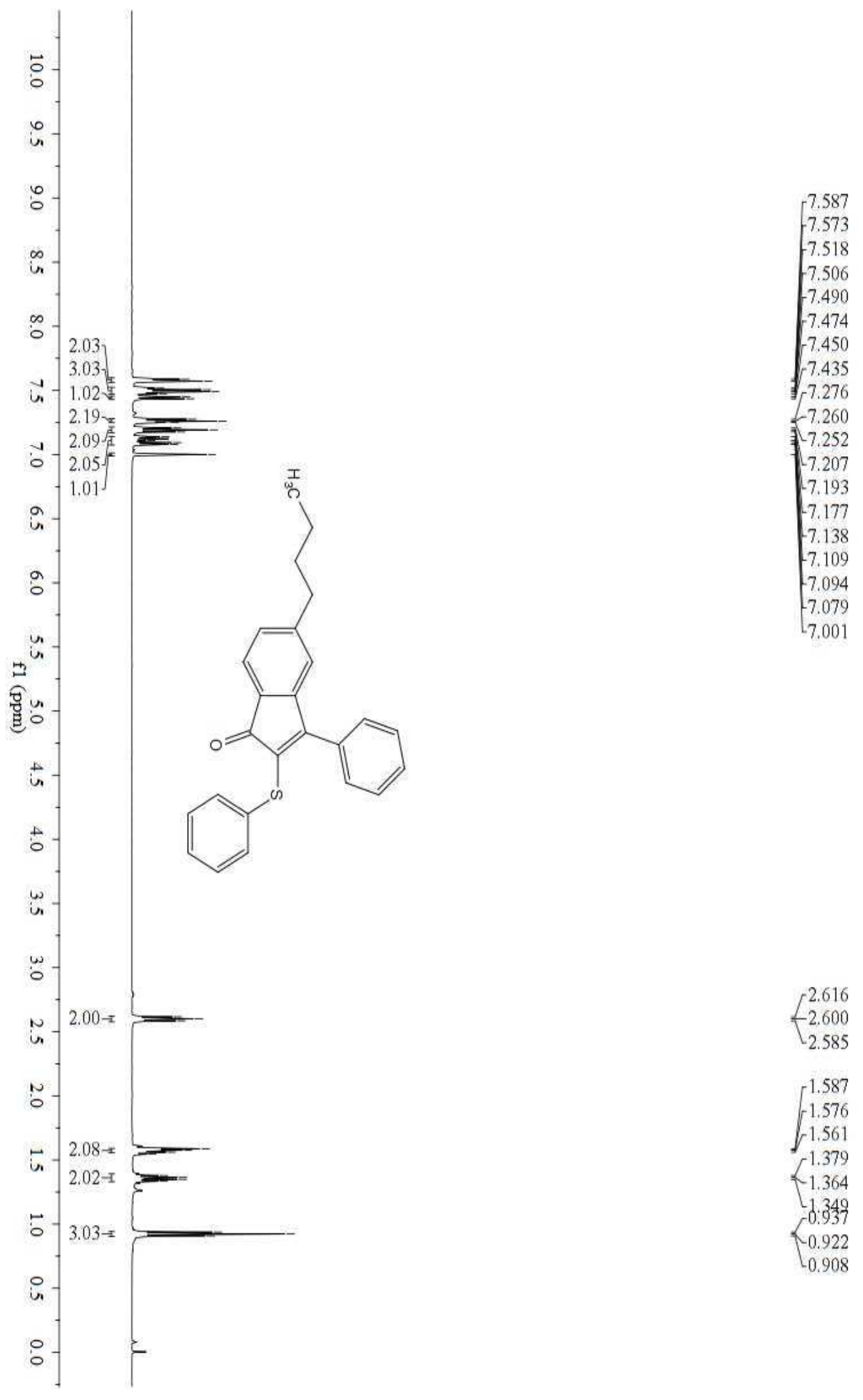


${ }^{13}$ C NMR of 5-butyl-3-phenyl-2-(phenylthio)-1H-inden-1-one (25)

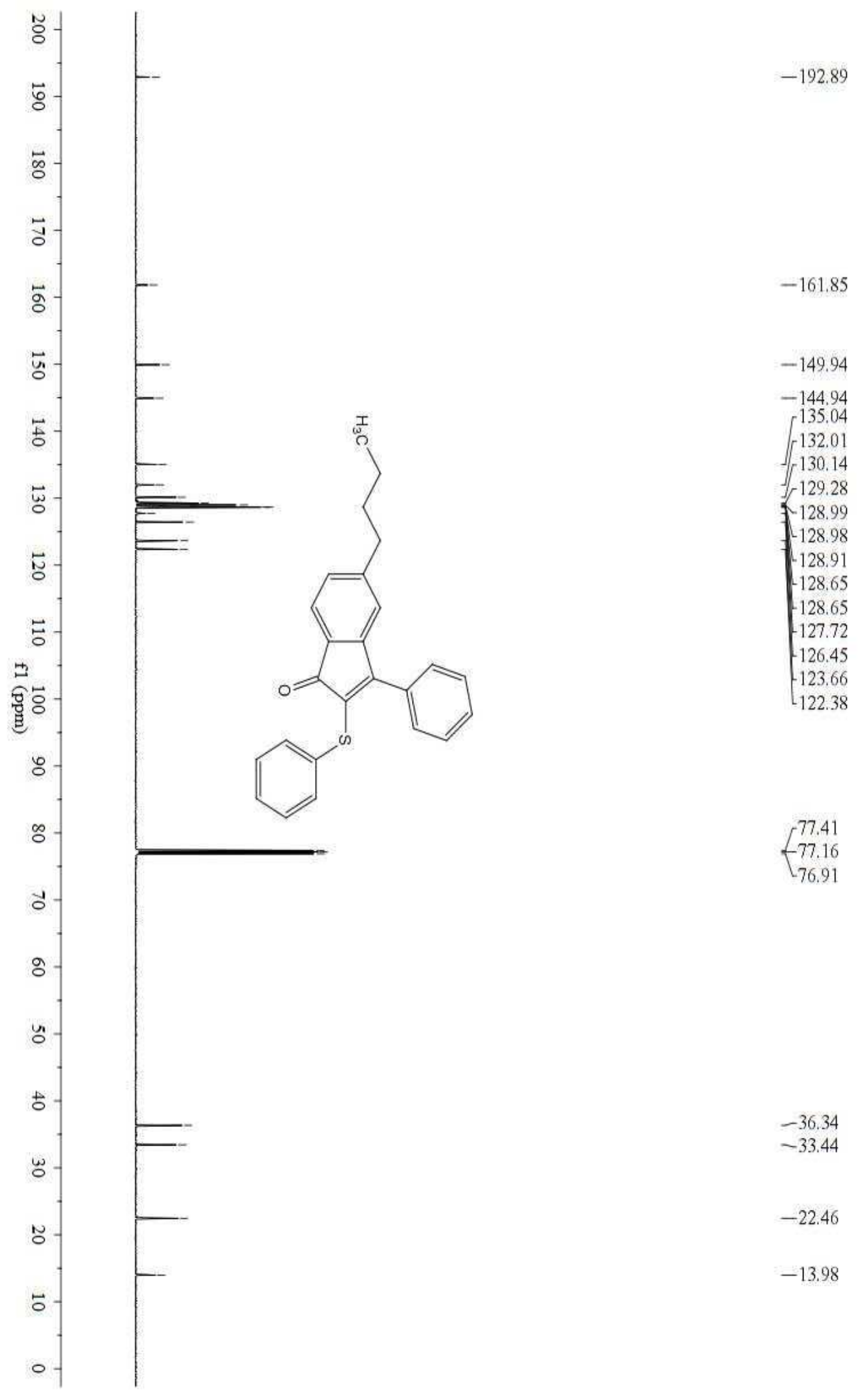




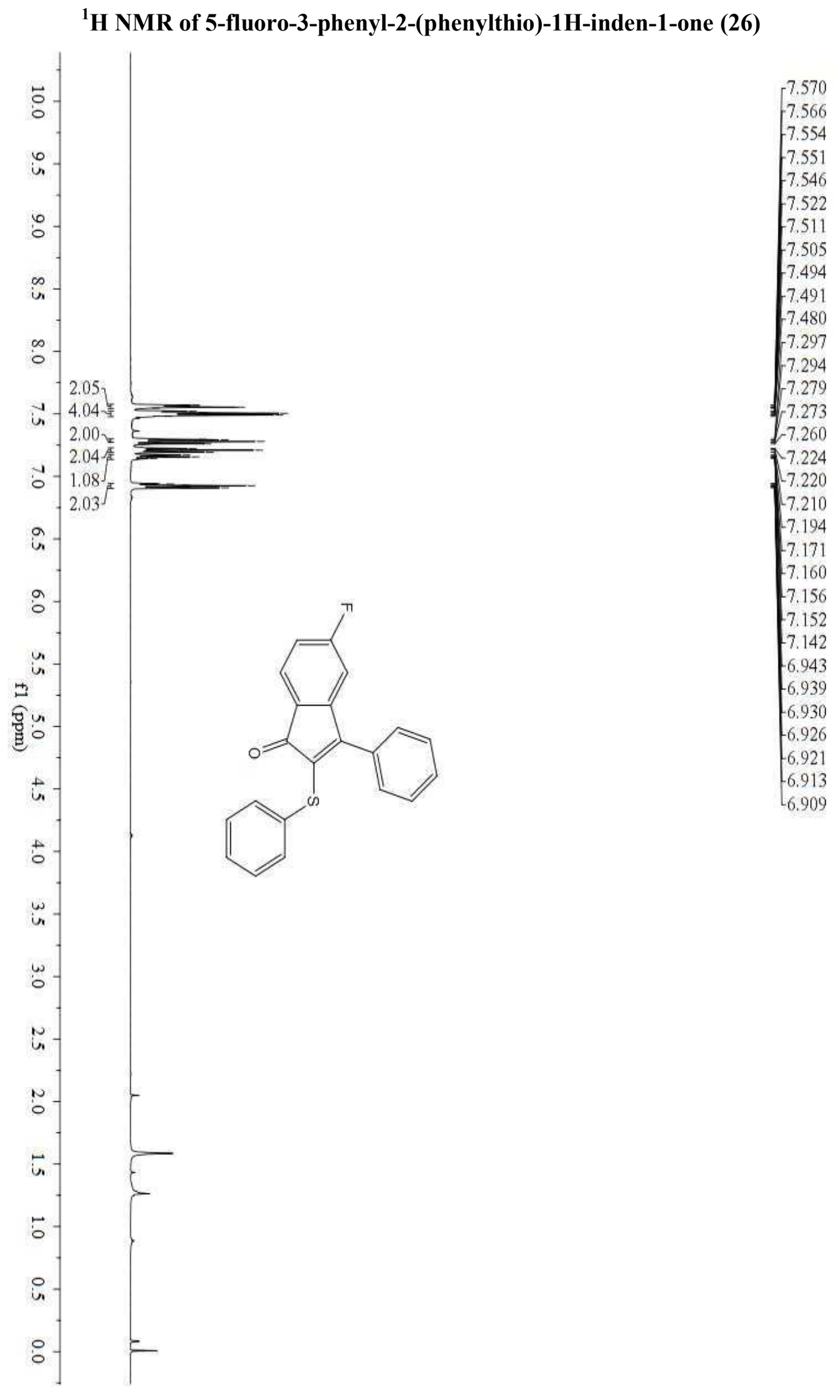


${ }^{13}$ C NMR of 5-fluoro-3-phenyl-2-(phenylthio)-1H-inden-1-one (26)

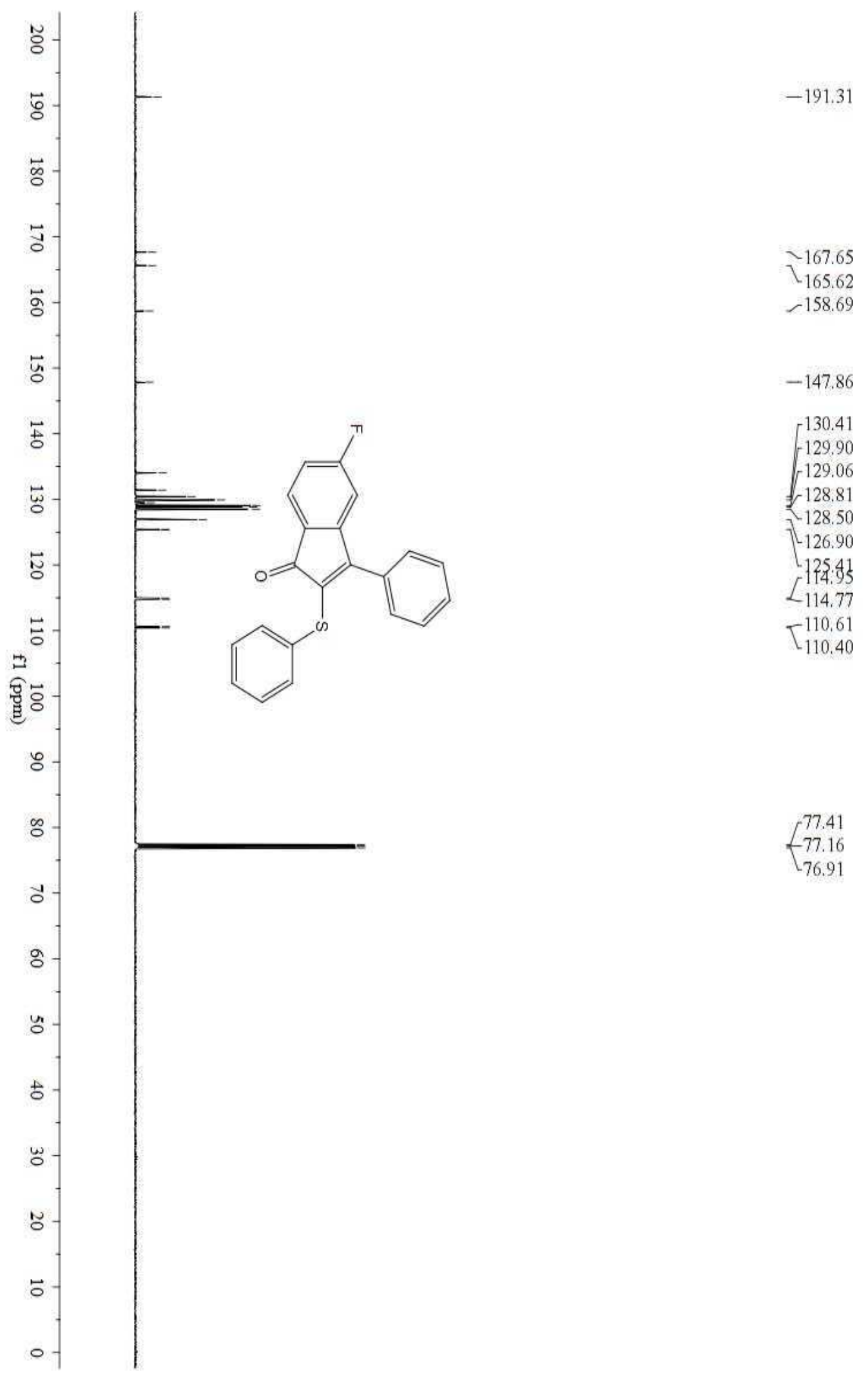




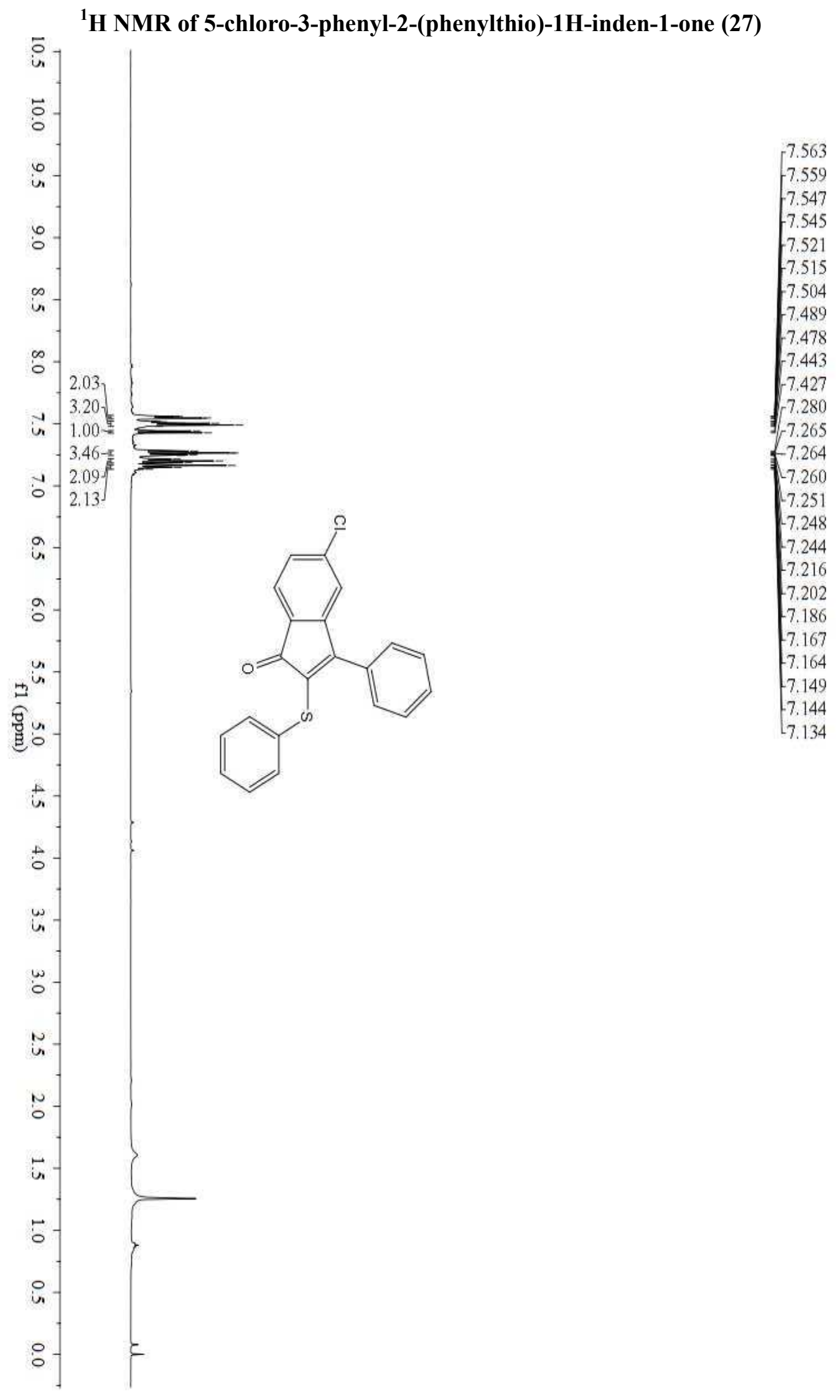


${ }^{13}$ C NMR of 5-chloro-3-phenyl-2-(phenylthio)-1H-inden-1-one (27)

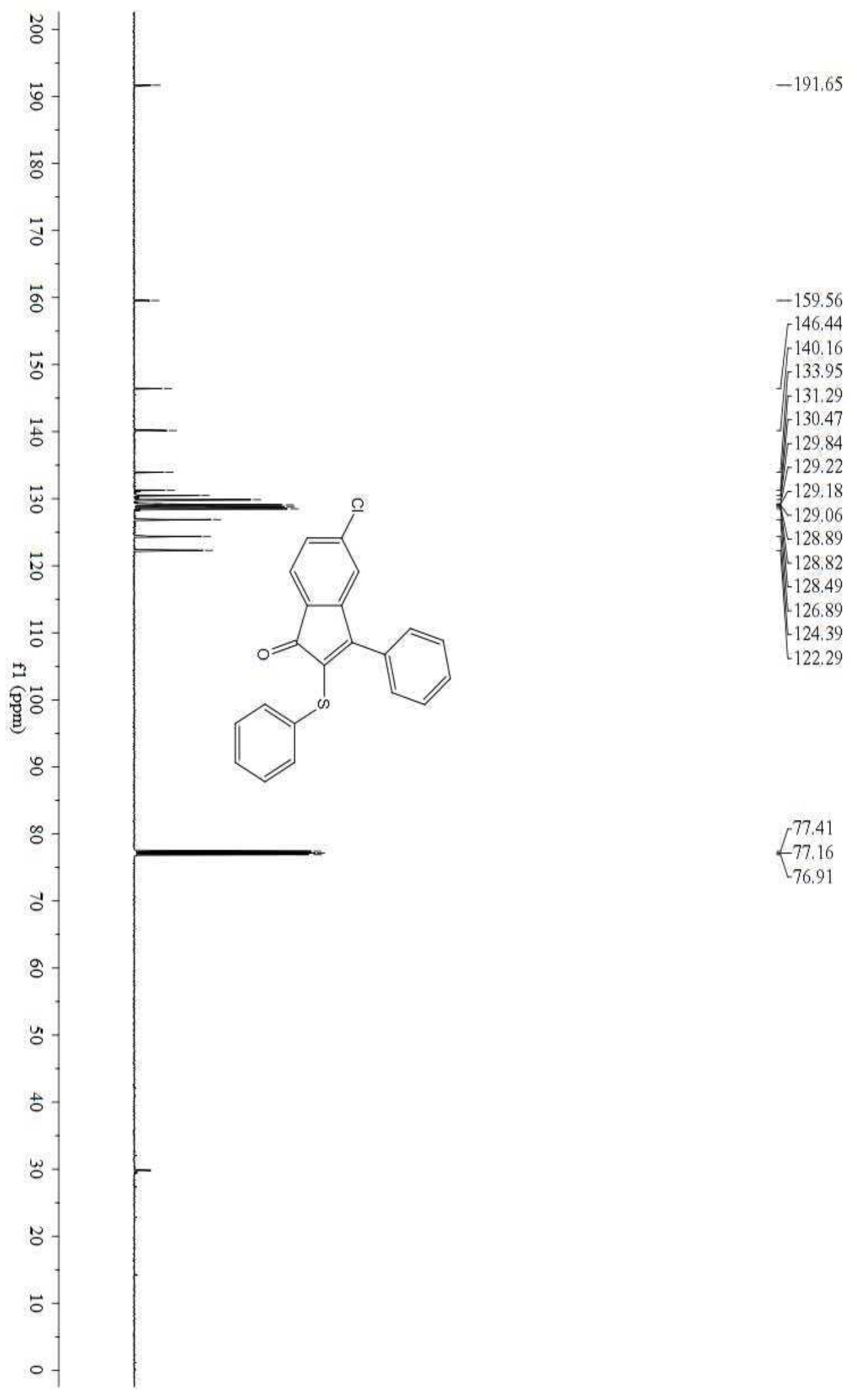


${ }^{1}$ H NMR of 5-bromo-3-phenyl-2-(phenylthio)-1H-inden-1-one (28)

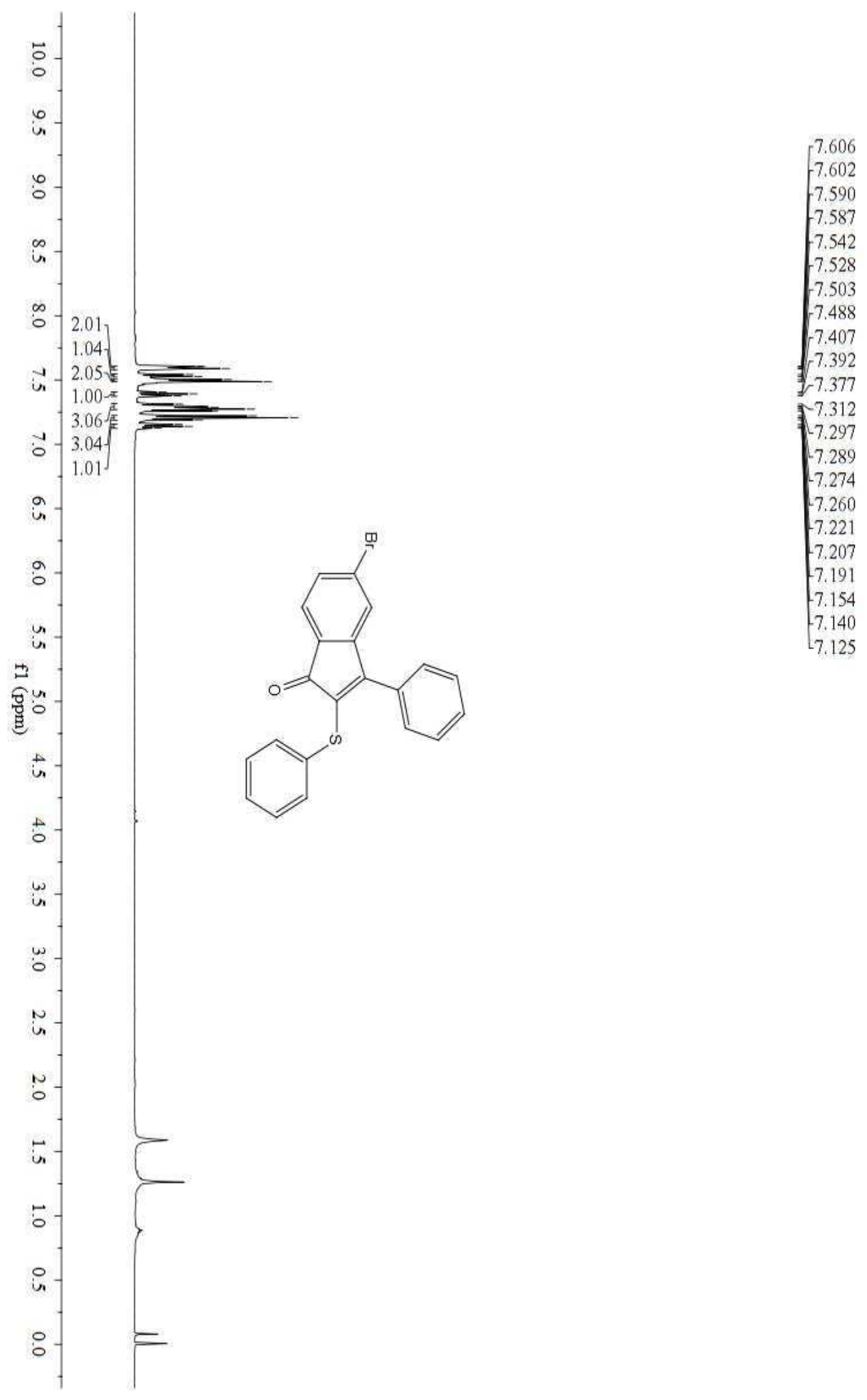


${ }^{13}$ C NMR of 5-bromo-3-phenyl-2-(phenylthio)-1H-inden-1-one (28)

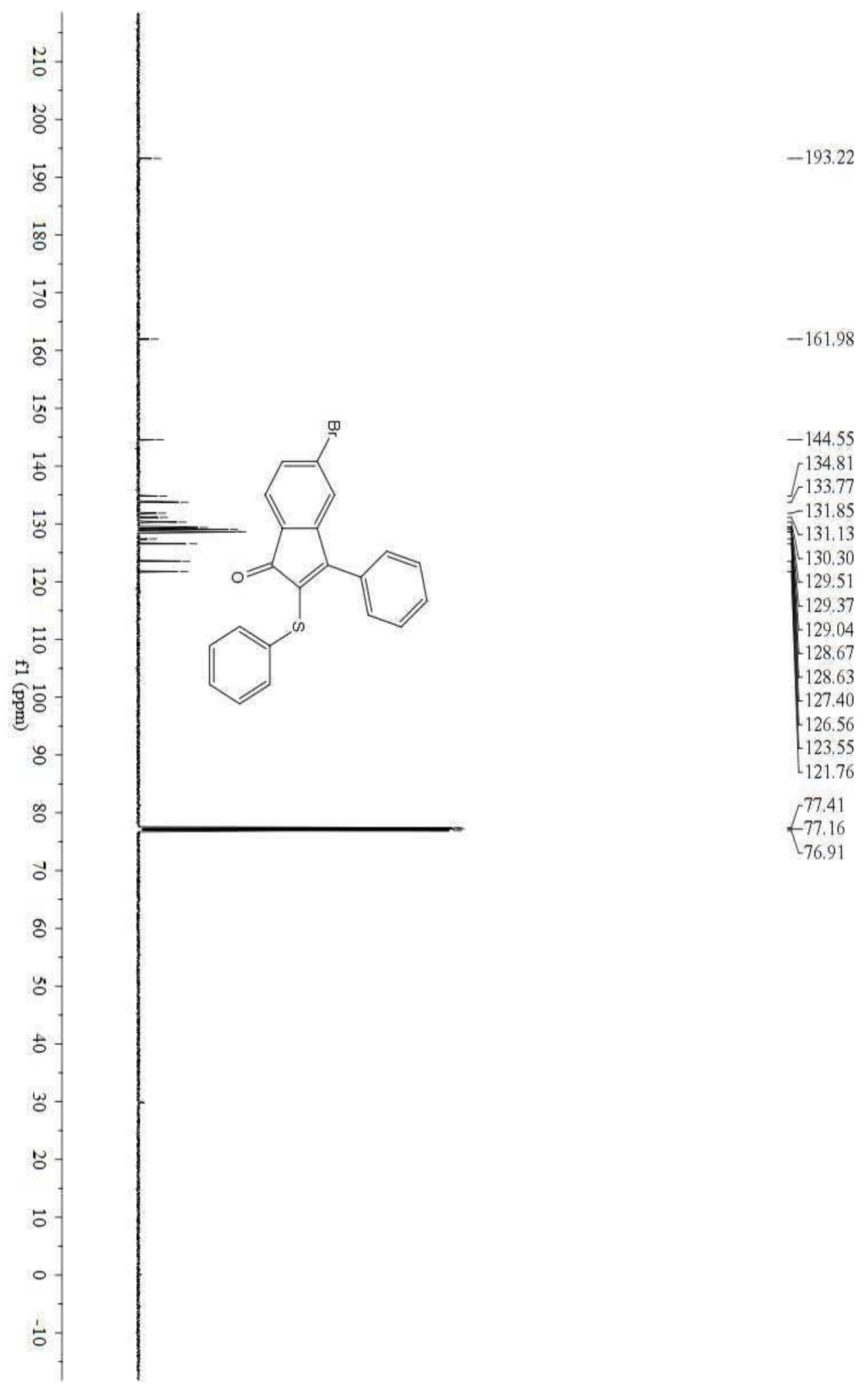


${ }^{1}$ H NMR of 6-chloro-3-phenyl-2-(phenylthio)-1H-inden-1-one (29a)

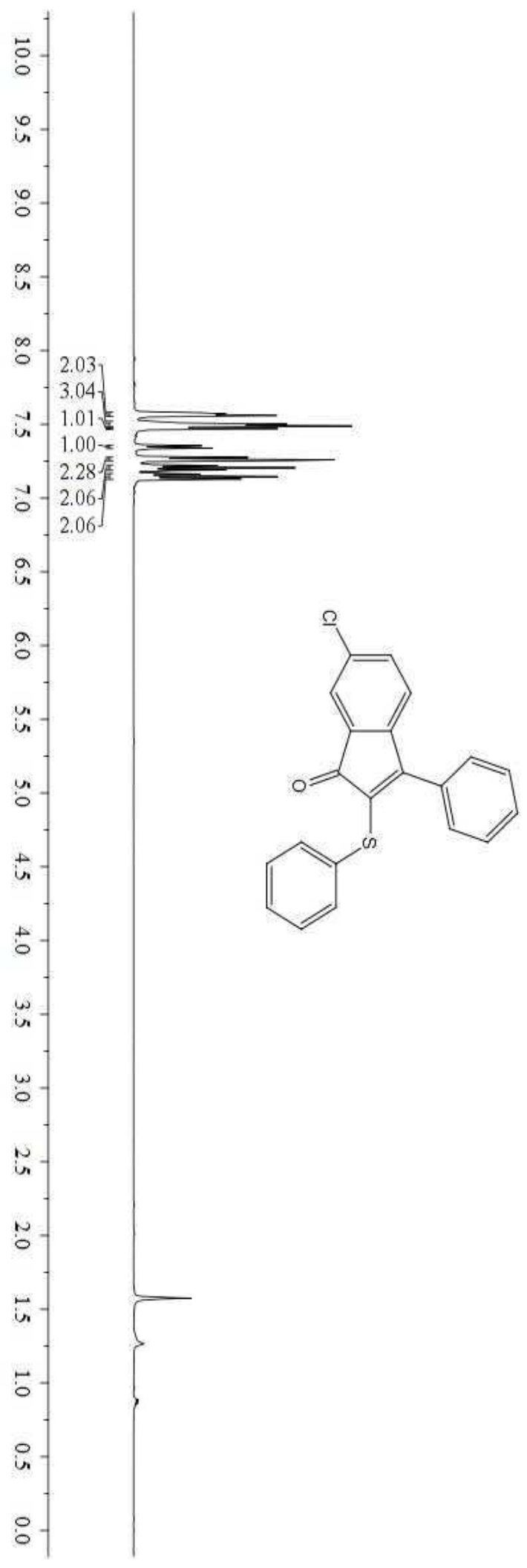

7.191

$-7.163$

$-7.145$

$-7.129$ 
${ }^{13}$ C NMR of 4-chloro-3-phenyl-2-(phenylthio)-1H-inden-1-one (29a)

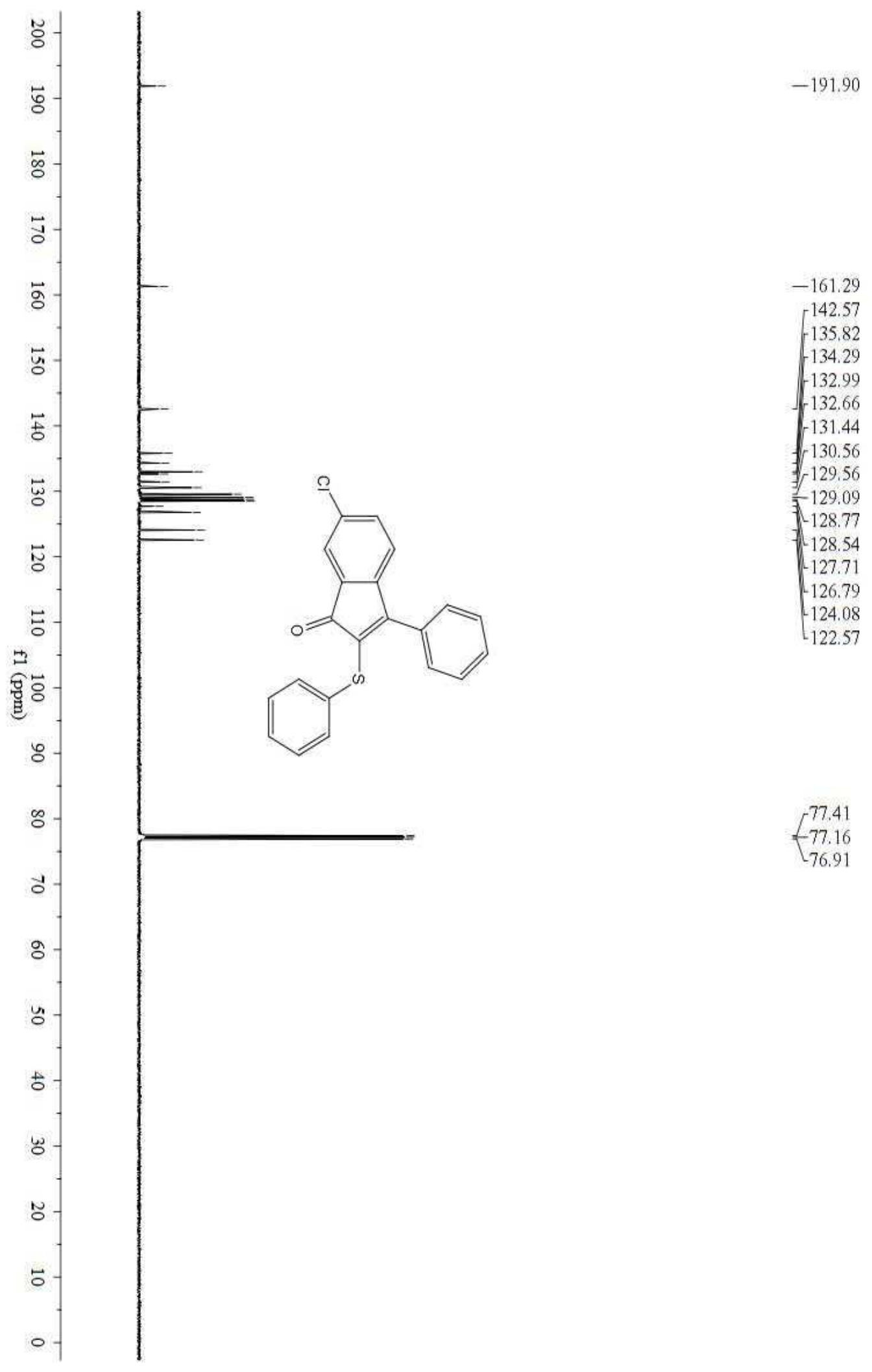


${ }^{1}$ H NMR of 4-chloro-3-phenyl-2-(phenylthio)-1H-inden-1-one (29b)

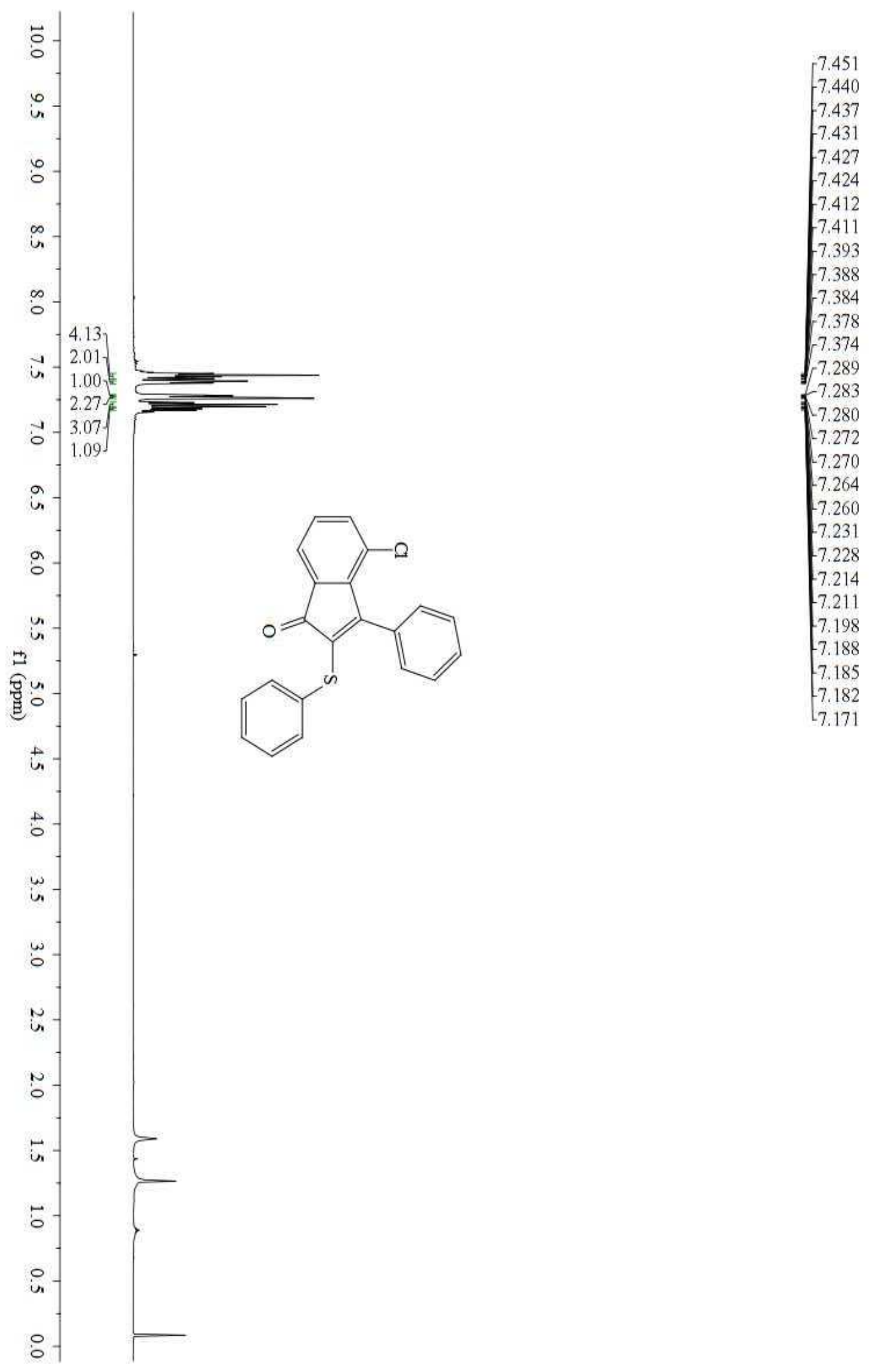


${ }^{13}$ C NMR of 4-chloro-3-phenyl-2-(phenylthio)-1H-inden-1-one (29b)

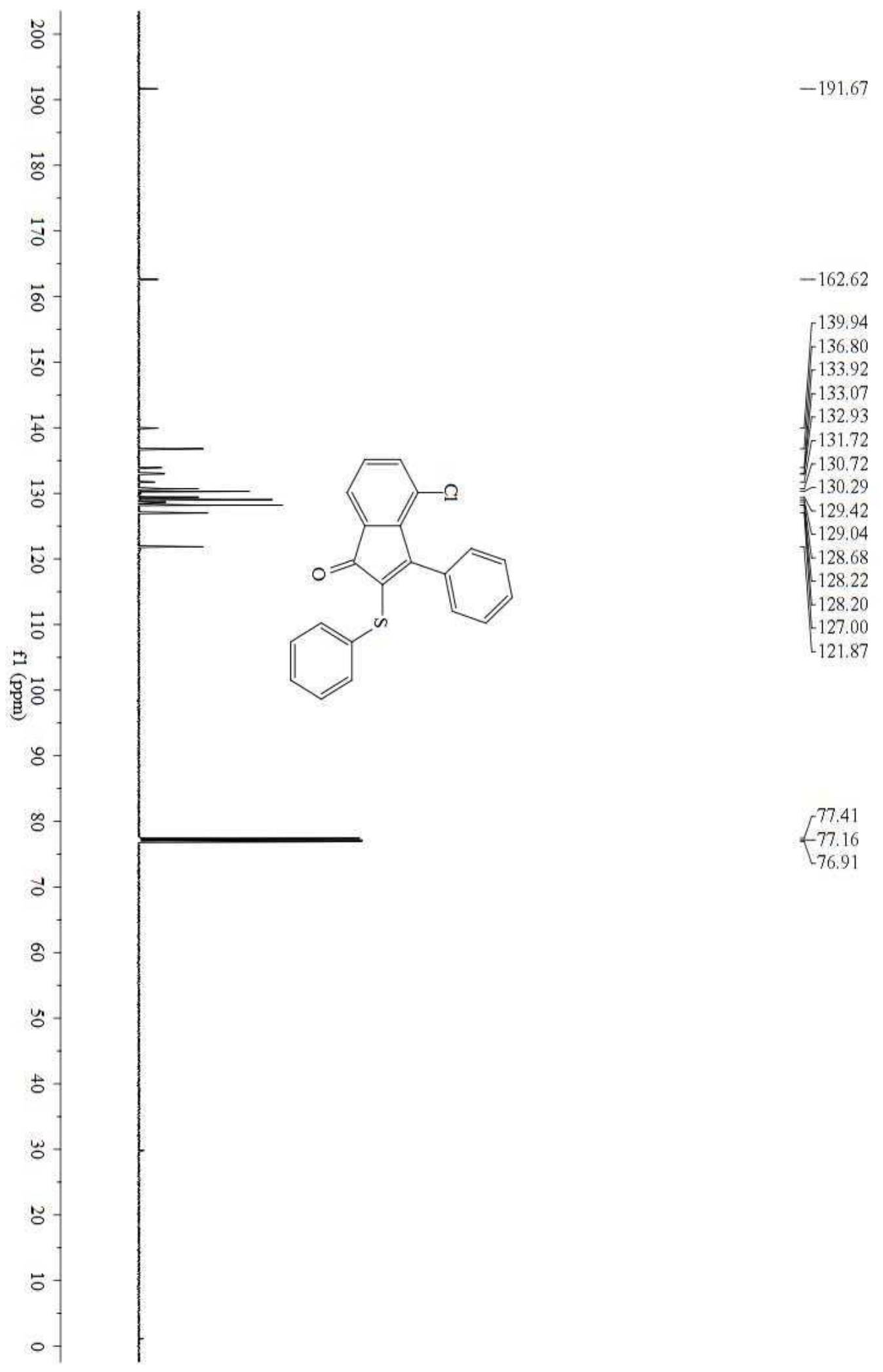




\section{X-Ray of compound 16}

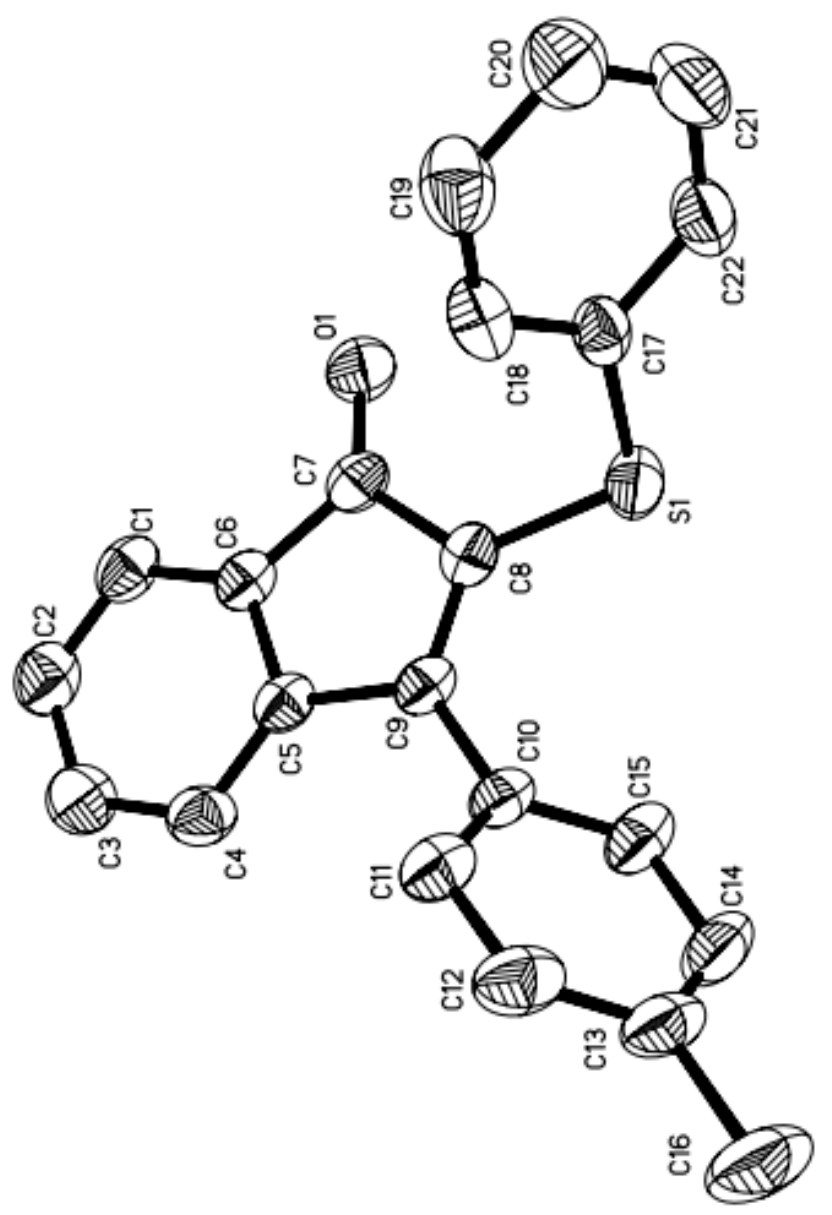

The displacement ellipsoids are drawn at the $30 \%$ probability level.

Single crystals suitable for X-ray analysis were obtained by slow evaporation of the mixed solution of petroleum ether (bp $60-90{ }^{\circ} \mathrm{C}$ ) and trace amount of EtOAc. 
Table S1: Crystal Structure and Data Refinement Parameters of compound 16

\begin{tabular}{|l|l|}
\hline Compound & \\
\hline Empirical Formula & $\mathrm{C}_{22} \mathrm{H}_{16} \mathrm{O} \mathrm{S}$ \\
\hline Formula Weight & 328.41 \\
\hline Crystal System / Space Group & Monoclinic, -P 21/c \\
\hline $\mathrm{a} / \AA$ & $10.168(3)$ \\
\hline $\mathrm{b} / \AA$ & $15.700(4)$ \\
\hline $\mathrm{c} / \AA$ & $11.009(3)$ \\
\hline$\alpha /{ }^{\circ}$ & 90 \\
\hline$\beta /{ }^{\circ}$ & $94.542(5)$ \\
\hline$\gamma /{ }^{\circ}$ & 90 \\
\hline $\mathrm{V} / \AA^{3}$ & $1752.1(8)$ \\
\hline $\mathrm{Z}$ & 4 \\
\hline $\mathrm{D}$ calc $\left(\mathrm{g} / \mathrm{cm}^{3}\right)$ & 1.245 \\
\hline$\mu\left(\mathrm{mm}{ }^{-1}\right)$ & 0.189 \\
\hline Crystal size (mm) & $0.36 \times 0.27 \mathrm{x} 0.25$ \\
\hline Color / Shape & white \\
\hline Temp (K) & $298(2)$ \\
\hline Theta range for collection & 2.01 to 26.00 \\
\hline Reflections collected & 14037 \\
\hline Independent reflections & 3441 \\
\hline Data/restraints/parameters & $3441 / 0 / 218$ \\
\hline Goodness of fit on $\mathrm{F}^{2}$ & 1.045 \\
\hline Final R indices $[\mathrm{I}>2 \sigma(\mathrm{I})]$ & $\mathrm{R} 1=0.0422, \mathrm{wR} 2=0.1305$ \\
\hline $\mathrm{R}$ indices (all data) & $\mathrm{R} 1=0.0618, \mathrm{wR} 2=0.1417$ \\
\hline Largest difference peak/hole & 0.168 and -0.152 \\
\hline & \\
\hline
\end{tabular}

